\title{
PERTURBED CLOSED GEODESICS ARE PERIODIC ORBITS: INDEX AND TRANSVERSALITY
}

\author{
JOA WEBER
}

\begin{abstract}
We study the classical action functional $\mathcal{S}_{V}$ on the free loop space of a closed, finite dimensional Riemannian manifold $M$ and the symplectic action $\mathcal{A}_{V}$ on the free loop space of its cotangent bundle. The critical points of both functionals can be identified with the set of perturbed closed geodesics in $M$. The potential $V \in C^{\infty}\left(M \times S^{1}, \mathbb{R}\right)$ serves as perturbation and we show that both functionals are Morse for generic $V$. In this case we prove that the Morse index of a critical point $x$ of $\mathcal{S}_{V}$ equals minus its Conley-Zehnder index when viewed as a critical point of $\mathcal{A}_{V}$ and if $x^{*} T M \rightarrow S^{1}$ is trivial. Otherwise a correction term +1 appears.
\end{abstract}

\section{Contents}

1. Introduction and main results 1

2. The index theorem 5

2.1. The Hessians 5

$\begin{array}{lll}2.2 . & \text { Orthonormal and unitary trivializations } & 6\end{array}$

2.3. Morse index 8

$\begin{array}{lll}2.4 . & \text { Conley-Zehnder index } & 8\end{array}$

$\begin{array}{lll}2.5 . & \text { Robbin-Salamon index } & 11\end{array}$

$\begin{array}{lll}2.6 . & \text { Spectral flow and Conley Zehnder index } & 13\end{array}$

$\begin{array}{lll}2.7 . & \text { Proof of the index theorem 1.2 } & 19\end{array}$

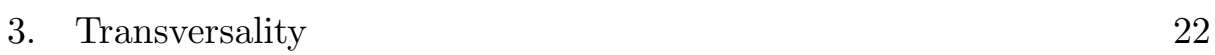

3.1. Thom-Smale transversality 22

3.2. Transversality in the $C^{k}$-category $\quad 25$

3.3. Transversality in the $C^{\infty}$-category 29

\begin{tabular}{ll|l}
\hline Appendix A. Finite Sum & 31
\end{tabular}

References 32

\section{InTRODUCTION AND MAIN RESUltS}

We consider a smooth, compact Riemannian manifold $M$ of dimension $n$ and without boundary. The inner product on the tangent space $T_{x} M$ is denoted by $\langle\cdot, \cdot\rangle$ and $g(x): T_{x} M \rightarrow T_{x}^{*} M$ is the induced isomorphism. We

\footnotetext{
${ }^{0}$ Mathematics Subject Classification (2000) 53-01, 53D25, 53D12, 53C22, 37J45

Date: October 29, 2018.
} 
study the set of critical points of the classical action functional $\mathcal{S}_{V}$ on the space $\mathcal{L} M$ of free smooth loops in $M$ which is defined by

$$
\mathcal{S}_{V}(x):=\int_{0}^{1} L(t, x(t), \dot{x}(t)) d t
$$

The Lagrangian function $L=L_{V}: S^{1} \times T M \rightarrow \mathbb{R}$ has the special form kinetic minus potential energy

$$
L(t, x(t), \dot{x}(t)):=\frac{1}{2}|\dot{x}(t)|^{2}-V(t, x(t))
$$

with a time periodic potential $V \in C^{\infty}\left(S^{1} \times M, \mathbb{R}\right)$. Here and throughout we identify $S^{1}$ with $\mathbb{R} / \mathbb{Z}$ and represent the loop $x \in \mathcal{L} M$ as a smooth periodic function $x: \mathbb{R} \rightarrow M$ satisfying $x(t+1)=x(t)$. The $L^{2}$-gradient of $\mathcal{S}_{V}$ is easily computed to be

$$
\operatorname{grad} \mathcal{S}_{V}(x)=-\nabla_{t} \dot{x}-\nabla V(t, x)
$$

where $\nabla V$ denotes the gradient of $V$ with respect to the $x$-variable and where $\nabla_{t}$ denotes covariant differentiation in direction $\dot{x}$ with respect to the Levi-Civita connection $\nabla$. The set of critical points of $\mathcal{S}_{V}$ is abbreviated by

$$
\text { Crit }=\text { Crit } \mathcal{S}_{V}=\left\{x \in C^{\infty}\left(S^{1}, M\right) \mid-\nabla_{t} \dot{x}-\nabla V(t, x)=0\right\}
$$

We call its elements perturbed closed geodesics, since it coincides with the set of closed geodesics on $M$ in the special case of constant potential $V$.

On the other hand the symplectic action functional $\mathcal{A}_{V}$ on the free loop space $\mathcal{L} T^{*} M$ of $T^{*} M$ is defined as

$$
\mathcal{A}_{V}(z):=\int_{0}^{1}(\langle y(t), \dot{x}(t)\rangle-H(t, z(t))) d t
$$

where $z=(x, y)$ and the time dependent Hamiltonian function $H=H_{V}$ : $S^{1} \times T^{*} M \rightarrow \mathbb{R}$ is the Legendre transform of $L$; namely kinetic plus potential energy

$$
H(t, x, y)=\frac{1}{2}|y|^{2}+V(t, x), \quad(x, y) \in T^{*} M
$$

The set $C r i t \mathcal{A}_{V}$ of critical points of $\mathcal{A}_{V}$ can be naturally identified with the set $\operatorname{Crit} \mathcal{S}_{V}$ via the bijection

$$
\text { Crit } \mathcal{S}_{V} \rightarrow \text { Crit } \mathcal{A}_{V}: x \mapsto z_{x}=(x, g(x) \dot{x})
$$

The inverse map is given by projecting onto $M$. Note that $\mathcal{S}_{V}(x)=\mathcal{A}_{V}\left(z_{x}\right)$ for $x \in$ Crit.

Our first result asserts that the functionals $\mathcal{S}_{V}$ and $\mathcal{A}_{V}$ are Morse for generic $V$. More precisely, in subsection 2.1 it will turn out that the Hessian of $\mathcal{S}_{V}$ at a critical point $x$ can be represented by the perturbed Jacobi operator $A_{x}$ in $L^{2}\left(S^{1}, x^{*} T M\right)$ with dense domain $W^{2,2}\left(S^{1}, x^{*} T M\right)$ given by

$$
A_{x} \xi=-\nabla_{t} \nabla_{t} \xi-R(\xi, \dot{x}) \dot{x}-\nabla_{\xi} \nabla V_{t}(x)
$$


and where $R$ denotes the Riemann curvature tensor. This operator is injective if and only if it is surjective. A critical point $x$ of $\mathcal{S}_{V}$ is called nondegenerate if $A_{x}$ is injective. A function with nondegenerate critical points only is called a Morse function. For $a \in \mathbb{R}$ let

$$
\mathcal{L}^{a} M:=\mathcal{L} M \cap \mathcal{S}_{V}^{-1}(-\infty, a], \quad \operatorname{Crit}^{a}:=\operatorname{Crit} \cap \mathcal{L}^{a} M
$$

The space $\mathcal{L}^{a} T^{*} M$ is defined similarly with $\mathcal{S}_{V}$ replaced by $\mathcal{A}_{V}$. Define the set $\mathcal{V}_{\text {reg }}$ of regular potentials to be the set of all $V \in C^{\infty}\left(S^{1} \times M, \mathbb{R}\right)$ such that $\mathcal{S}_{V}: \mathcal{L} M \rightarrow \mathbb{R}$ is Morse. Restricting $\mathcal{S}_{V}$ to $\mathcal{L}^{a} M$ we obtain the set

$$
\mathcal{V}_{r e g}^{a}:=\left\{V \in C^{\infty}\left(S^{1} \times M, \mathbb{R}\right) \mid A_{x} \text { is surjective } \forall x \in C r i t^{a}\right\}
$$

In subsection 2.1 we shall see that a critical point $x$ of $\mathcal{S}_{V}$ is nondegenerate if and only if the corresponding critical point $z_{x}$ of $\mathcal{A}_{V}$ is. In other words $\mathcal{S}_{V}$ is Morse if and only if $\mathcal{A}_{V}$ is.

Theorem 1.1. (Transversality) i) For every $a \in \mathbb{R}$ the subset $\mathcal{V}_{\text {reg }}^{a} \subset$ $C^{\infty}\left(S^{1} \times M, \mathbb{R}\right)$ of regular potentials is open and dense.

ii) The subset of regular potentials $\mathcal{V}_{\text {reg }} \subset C^{\infty}\left(S^{1} \times M, \mathbb{R}\right)$ is residual and therefore dense.

The set $C^{\infty}\left(S^{1} \times M, \mathbb{R}\right)$ is a complete metric space with the distance function

$$
d\left(V_{1}, V_{2}\right):=\sum_{k=0}^{\infty} \frac{1}{2^{k}} \frac{\left\|V_{1}-V_{2}\right\|_{C^{k}}}{1+\left\|V_{1}-V_{2}\right\|_{C^{k}}}
$$

A residual set is by definition one which contains a countable intersection of open and dense sets. According to Baire's category theorem [RS80, section III.5] such a set is dense.

Choosing a regular potential $V$, we can assign two integers to any $x \in C$ rit as follows: On the one hand the perturbed closed geodesic $x$ has a Morse index $\operatorname{Ind}(x)$, namely the number of negative eigenvalues of $A_{x}$ counted with multiplicities, and on the other hand it is possible to interpret $z_{x}$ as a periodic orbit of the Hamiltonian system on $T^{*} M$ with Hamiltonian (2) and therefore define its Conley-Zehnder index $\mu_{C Z}\left(z_{x}\right)$. Since there exists a global Lagrangian splitting of $T\left(T^{*} M\right)$, the latter is well defined, at least if $x^{*} T M \rightarrow S^{1}$ is trivial. Otherwise there is some choice involved which leads to well definedness modulo 2 only. Our second result relates both indices.

Theorem 1.2. (Index) Let $x \in C r i t$ be nondegenerate, then

$$
\mu_{C Z}\left(z_{x}\right)=-\operatorname{Ind}(x)
$$

if the bundle $x^{*} T M \rightarrow S^{1}$ is trivial and

$$
\mu_{C Z}\left(z_{x}\right)=-\operatorname{Ind}(x)+1
$$

if the bundle $x^{*} T M \rightarrow S^{1}$ is nontrivial.

The relation between the Maslov index and the Morse index of a closed geodesic has been studied first, as far as I know, by Duistermaat Du76. 
In the case of a closed geodesic on a flat torus theorem 1.2 had been obtained by Claude Viterbo Vi90 with a slightly different definition of the Conley-Zehnder index (apart from different normalizations): due to the degeneracy of his action functional he considered the Conley-Zehnder index of the linearized Hamiltonian flow on the energy surface restricted to directions normal to the trajectory.

A new feature arising in the present context is that $M$ is not required to be orientable and so $x^{*} T M \rightarrow S^{1}$ may not be trivial. On the other hand $z_{x}{ }^{*} T T^{*} M \rightarrow S^{1}$ always is. We overcome this problem by trivializing $x^{*} T M$ over $[0,1]$ and then apply to the induced trivialization of $z_{x}{ }^{*} T T^{*} M \rightarrow[0,1]$ an artificial half rotation to close up the frame and obtain a trivialization over $S^{1}$. Our choice of rotation is reflected in the formula by the term $\sigma(x)$. Other choices lead to other odd integer multiples of this term.

Application. Our results will be applied in We99, [SW01 and We01 to construct algebraic chain groups in the following way: Fix $a \in \mathbb{R}$, a regular potential $V$, and define

$$
C_{j}^{a}\left(M, g, V ; \mathbb{Z}_{2}\right):=\bigoplus_{\substack{x \in C r i t^{a} \\ I n d(x)=j}} Z_{2}\langle x\rangle
$$

Finiteness of the sum is a consequence of transversality combined with compactness as explained in appendix A. In case the negative gradient flows of $\mathcal{A}_{V}$ and $\mathcal{S}_{V}$ are Morse-Smale, we can count flow lines between critical points of index difference 1 to obtain chain maps $\partial^{F}$ and $\partial^{M}$, respectively. These flow lines are solutions of Floer's elliptic PDE on $T^{*} M$ and of the parabolic $L^{2}$-heat flow in the loop space of $M$, respectively. It is Floer's theorem [Fl89, Thm. 4] that $\partial^{F} \circ \partial^{F}=0$; up to the additional argument by Cieliebak [Ci94, theorem 5.4] in order to deal with noncompactness of $T^{*} M$. In a forthcoming paper We01 it will be shown that $\partial^{M}$ is a boundary operator too, whose homology represents the singular homology of $\mathcal{L}^{a} M$. In a joint research project with D. Salamon [SW01] we will show that both homology theories are naturally isomorphic

$$
H F_{*}^{a}\left(T^{*} M, H, J_{g} ; \mathbb{Z}_{2}\right) \simeq H M_{*}\left(\mathcal{S}_{V, g}, \mathcal{L}^{a} M ; \mathbb{Z}_{2}\right)
$$

As a consequence, the Floer homology of a cotangent bundle with a quadratic Hamiltonian of the form (2) is isomorphic to the singular homology of the loop space. This result has been obtained by Viterbo with different methods in [Vi96]. Our idea of proof is to obtain the heat equation as an adiabatic limit of Floer's elliptic PDE. The index theorem shows that the Morse index serves as a natural grading of Floer homology.

Remark 1.3. (Sign conventions and normalizations) The canonical symplectic structure $\omega_{\text {can }}$ on $T^{*} M$ is with respect to natural coordinates 
$\left(x^{1}, \ldots, x^{n}, y_{1}, \ldots, y_{n}\right)$ given by $\sum_{i=1}^{n} d x^{i} \wedge d y_{i}$. The standard complex structure $J_{0}$ on $\mathbb{R}^{2 n}$ and the signature of a symmetric matrix $S$ are defined by

$$
J_{0}:=\left(\begin{array}{cc}
0 & -\mathbb{1} \\
\mathbb{1} & 0
\end{array}\right), \quad \text { sign } S:=n^{+}(S)-n^{-}(S)
$$

where $n^{+(-)}(S)$ is the number of positive (negative) eigenvalues of $S$. The Conley-Zehnder index and the upward spectral flow are normalized as follows

$$
\mu_{C Z}\left(t \mapsto e^{-t J_{0} S}\right)=-\frac{1}{2} \operatorname{sign} S, \quad \mu_{\text {Spec }}(s \mapsto \arctan s)=1
$$

where $t \in[0,1], s \in \mathbb{R}$ and the symmetric matrix $S$ satisfies $\|S\|<2 \pi$.

Acknowledgements. The author is most grateful to Kai Cieliebak, Dietmar Salamon and Eduard Zehnder for stimulating discussions and comments.

\section{THE INDEX THEOREM}

2.1. The Hessians. The critical points $x$ of the classical action $\mathcal{S}_{V}$ are the solutions of the nonlinear equation

$$
-\nabla_{t} \partial_{t} x-\nabla V_{t}(x)=0
$$

which follows from the formula

$$
d \mathcal{S}_{V}(x) \xi=\int_{0}^{1}\left\langle-\nabla_{t} \dot{x}-\nabla V_{t}(x), \xi\right\rangle d t, \quad \forall \xi \in C^{\infty}\left(S^{1}, x^{*} T M\right)
$$

Linearization at a critical point $x$ leads to the Hessian of $\mathcal{S}_{V}$ at $x$ given by

$$
d^{2} \mathcal{S}_{V}(x)(\xi, \eta)=\left\langle A_{x} \xi, \eta\right\rangle_{L^{2}}=\int_{0}^{1}\left\langle-\nabla_{t} \nabla_{t} \xi-R(\xi, \dot{x}) \dot{x}-\nabla_{\xi} \nabla V_{t}(x), \eta\right\rangle d t
$$

In order to obtain this formula introduce local coordinates and a smooth variation $x_{\tau}=\left(x_{\tau}^{1}, \ldots, x_{\tau}^{n}\right)$ of $x$; i.e. $x_{\tau}$ depends smoothly on $\tau \in(-\delta, \delta)$, $\delta>0$ small, and $x_{0}=x,\left.\frac{d}{d \tau}\right|_{\tau=0} x_{\tau}=:\left(\xi^{1}, \ldots, \xi^{n}\right)$. In (5) replace $x$ by $x_{\tau}$, apply $\left.\frac{d}{d \tau}\right|_{\tau=0}$ and use the nonlinear equation as well as the representation of $R$ in local coordinates via the Christoffel symbols of $\nabla$ to obtain (3).

On the other hand the critical points of the symplectic action $\mathcal{A}_{V}$ correspond precisely to the 1-periodic solutions of the Hamiltonian system $\left(T^{*} M, \omega_{\text {can }}=-d \lambda, H_{V}\right)$. Here $\lambda$ is the Liouville form which, in natural coordinates $\left(x^{1}, \ldots, x^{n}, y_{1}, \ldots, y_{n}\right)$ of $T^{*} M$ introduced below, is given by $\sum_{i=1}^{n} y_{i} d x^{i}$. The Hamiltonian differential equation $\dot{z}(t)=X_{H_{V}}(t, z(t))$ can be expressed in the form

$$
\left(\begin{array}{c}
\partial_{t} x \\
\nabla_{t} y
\end{array}\right)=\left(\begin{array}{c}
g(x)^{-1} y \\
-g(x) \nabla V_{t}(x)
\end{array}\right)
$$

where $z(t)=(x(t), y(t))$ with $y(t) \in T_{x(t)}^{*} M$. Note that here and throughout we identify $T_{z(t)} T^{*} M$ with $T_{x(t)} M \oplus T_{x(t)}^{*} M$ by the isomorphism which takes $\partial_{t} z(t)$ to $\left(\partial_{t} x(t), \nabla_{t} y(t)\right)$. The equivalence of $(5)$ and $(6)$ is then obvious. In order to linearize (6) at a zero $(x, y)$ introduce local coordinates 
$\left(x^{1}, \ldots, x^{n}\right)$ for $x$ on $M$ and obtain natural coordinates for $y$ determined by $y=\sum_{j} y_{j} d x^{j}$. Choose a smooth variation $\left(x_{\tau}^{1}, \ldots, y_{n}^{\tau}\right)$ of $(x, y)$ and denote

$$
\left(\xi^{1}, \ldots, \xi^{n}, \tilde{\eta}_{1}, \ldots, \tilde{\eta}_{n}\right):=\left.\frac{d}{d \tau}\right|_{\tau=0}\left(\xi_{\tau}^{1}, \ldots, \xi_{\tau}^{n}, \tilde{\eta}_{1}^{\tau}, \ldots, \tilde{\eta}_{n}^{\tau}\right)
$$

It turns out that the $\xi^{k}$ transform as components of a vector $\xi \in T_{x} M$ under coordinate changes, but the $\tilde{\eta}_{\ell}$ do not have any global meaning. However, the following combination with the Christoffel symbols of the Levi-Civita connection $\nabla$

$$
\eta_{\ell}:=\tilde{\eta}_{\ell}-\Gamma_{i \ell}^{k}(x) \xi^{i} y_{k}, \quad \ell=1, \ldots, n
$$

represents a covector $\eta=\sum_{\ell} \eta_{\ell} d x^{\ell} \in T_{x}^{*} M$. Recall that $y_{k}$ denotes the $k^{\text {th }}$ component of the fibre part of the chosen zero $(x, y)$ of (6) with respect to natural coordinates. Now linearization of (6) at a solution $z_{x}=\left(x, g(x) \partial_{t} x\right)$ of (6) leads to the selfadjoint operator $A_{z_{x}}$ in $L^{2}\left(S^{1}, x^{*} T M \oplus x^{*} T^{*} M\right)$ with dense domain $W^{1,2}\left(S^{1}, x^{*} T M \oplus x^{*} T^{*} M\right)$ which represents the Hessian of $\mathcal{A}_{V}$ at $z_{x}$ and is given by

$$
A_{z_{x}}\left(\begin{array}{l}
\xi \\
\eta
\end{array}\right)=\left(\begin{array}{c}
-g^{-1}(x) \nabla_{t} \eta-R(\xi, \dot{x}) \dot{x}-\nabla_{\xi} \nabla V_{t}(x) \\
g(x) \nabla_{t} \xi-\eta
\end{array}\right)
$$

This operator is clearly injective if and only if $A_{x}$ is: a short calculation shows that $\left(\xi, g(x) \nabla_{t} \xi\right) \in \operatorname{ker} A_{z_{x}}$ if and only if $\xi \in \operatorname{ker} A_{x}$ and this proves

Lemma 2.1. $\mathcal{A}_{V}$ is a Morse function if and only if $\mathcal{S}_{V}$ is.

2.2. Orthonormal and unitary trivializations. In order to compare the Morse index of a critical point $x$ of $\mathcal{S}_{V}$ and the Conley-Zehnder index of the critical point $z_{x}$ of $\mathcal{A}_{V}$ it will be convenient to view $x^{*} T M$ as vector bundle over $[0,1]$, choose an orthonormal trivialization $\phi$ satisfying a certain boundary condition and express the perturbed Jacobi operator $A_{x}$ with respect to this orthonormal frame. This same frame $\phi$ will then be used to construct a unitary trivialization $\Phi_{U}$ for the trivial bundle $x^{*} T M \oplus x^{*} T^{*} M \rightarrow S^{1}$. In case $x^{*} T M \rightarrow S^{1}$ is nontrivial some additional half rotation $U$ has to be applied in order to obtain indeed a frame over $S^{1}$ rather than [0,1]. More precisely, let

$$
\sigma=\sigma(x):= \begin{cases}0 & \text { if } x^{*} T M \text { is trivial } \\ 1 & \text { otherwise }\end{cases}
$$

and

$$
E_{\sigma}:=\operatorname{diag}_{n}\left((-1)^{\sigma}, 1, \ldots, 1\right) \in \mathbb{R}^{n \times n}
$$

Since $O(n)$ has precisely two connected components one of which contains $E_{0}$ and the other one $E_{1}$, we may choose an orthonormal trivialization

$$
\phi=\phi_{\sigma}:[0,1] \times \mathbb{R}^{n} \rightarrow x^{*} T M
$$

such that $\phi(1)=\phi(0) E_{\sigma}$. Let $\dot{\xi}$ denote $\partial_{t} \xi$, define the space

$$
C_{\sigma}^{\infty}\left([0,1], \mathbb{R}^{n}\right):=\left\{\xi \in C^{\infty}\left([0,1], \mathbb{R}^{n}\right) \mid \xi(1)=E_{\sigma} \xi(0), \dot{\xi}(1)=E_{\sigma} \dot{\xi}(0)\right\}
$$


and let $W_{\sigma}^{2,2}=W_{\sigma}^{2,2}\left([0,1], \mathbb{R}^{n}\right)$ and $L_{\sigma}^{2}=L_{\sigma}^{2}\left([0,1], \mathbb{R}^{n}\right)$ be the closure of $C_{\sigma}^{\infty}\left([0,1], \mathbb{R}^{n}\right)$ with respect to the Sobolev $W^{2,2}$ - and $L^{2}$-norms, respectively.

We define the model operator $A^{0}: L_{\sigma}^{2} \supset W_{\sigma}^{2,2} \rightarrow L_{\sigma}^{2}$ for $A_{x}$ with respect to the isometry induced by $\phi$ by

$$
A^{0} \xi:=\phi^{-1} A_{x} \phi \xi=-\partial_{t} \partial_{t} \xi-B \xi-Q \xi
$$

Induced covariant differentiation in direction $\dot{x}$ in the trivial bundle $[0,1] \times \mathbb{R}^{n}$ is of the form

$$
\phi^{-1}(t) \nabla_{t} \phi(t) \xi(t)=\partial_{t} \xi(t)+P(t) \xi(t)
$$

where the connection potential $P$ is a family of skewsymmetric matrices. Then the $L^{2}$-symmetric first order operator $B$ and the family of symmetric matrices $Q$ in (9) are given by

$$
\begin{gathered}
B \xi=\left(\partial_{t}+P\right)^{2} \xi-\partial_{t} \partial_{t} \xi=2 P \partial_{t} \xi+\left(\partial_{t} P\right) \xi+P^{2} \xi \\
Q \xi=\phi^{-1}\left(R(\phi \xi, \dot{x}) \dot{x}+\nabla_{\phi \xi} \nabla V_{t}(x)\right)
\end{gathered}
$$

For $t \in \mathbb{R}$ let now $U(t)$ be the $2 n \times 2 n$ matrix which represents rotation of the $(1, n+1)$-coordinate plane by the angle $\pi t$ and is the identity on all other coordinates

$$
U(t)=\left(\begin{array}{cc}
\operatorname{diag}_{n}(\cos \pi t, 1, \ldots, 1) & \operatorname{diag}_{n}(-\sin \pi t, 0, \ldots, 0) \\
\operatorname{diag}_{n}(\sin \pi t, 0, \ldots, 0) & \operatorname{diag}_{n}(\cos \pi t, 1, \ldots, 1)
\end{array}\right)
$$

The orthonormal trivialization $\phi=\phi_{\sigma}$ introduced above leads to a unitary trivialization of $x^{*} T M \oplus x^{*} T^{*} M$ over [0,1] only, namely

$$
\Phi=\Phi_{\sigma}:=\left(\begin{array}{cc}
\phi_{\sigma} & 0 \\
0 & \phi_{\sigma}^{*-1}
\end{array}\right)
$$

Multiplication by $U(t) \in S p(2 n) \cap O(2 n)$ then gives rise to a unitary trivialization of $x^{*} T M \oplus x^{*} T^{*} M$ over $S^{1}$

$$
\Phi_{U}=\Phi_{U, \sigma}:=\Phi_{\sigma} U^{-\sigma}: S^{1} \times \mathbb{R}^{2 n} \rightarrow x^{*} T M \oplus x^{*} T^{*} M
$$

In case $\sigma(x)=0, U^{-\sigma}=\mathbb{1}$ takes no effect. This is fine since we already have $\Phi(1)=\Phi(0)$, because of $\phi(1)=\phi(0)$. Otherwise multiplication by $U^{-1}$ serves to close up the frame at $t=1$. We may assume without loss of generality that the frame closes up smoothly: If not, we modify $\phi$ such that $\nabla_{t} \phi=0$ near the ends of $[0,1]$. Of course we could choose in (12) instead of $U$ some other power $U^{2 k+1}$, where $k \in \mathbb{Z}$ determines direction and multiplicity of rotation. This would lead to a change of the ConleyZehnder index in definition 2.3 by $2 k \sigma(x)$ as stated in lemma 2.4 (ii).

Finally, the operator $A_{z_{x}}$ is represented with respect to the unitary frame $\Phi_{U}$ by the linear operator $A^{1}$ in $L^{2}\left(S^{1}, \mathbb{R}^{2 n}\right)$ with dense domain $W^{1,2}\left(S^{1}, \mathbb{R}^{2 n}\right)$. Calculation leads to

$$
A^{1}:=\Phi_{U}^{-1} A_{z_{x}} \Phi_{U}=J_{0} \partial_{t}-S_{U}
$$

where

$$
S_{U}=U^{\sigma} S U^{-\sigma}-J_{0} U^{\sigma} \partial_{t}\left(U^{-\sigma}\right), \quad S=\left(\begin{array}{cc}
Q & P \\
-P & \mathbb{1}
\end{array}\right)
$$


and $Q, P$ are the families of matrices in (9) and (10), respectively. It is easy to check that $S$ is a family of symmetric matrices and $S_{U}$ is symmetric with respect to the $L^{2}$-inner product.

2.3. Morse index. Let $x$ be a critical point of $\mathcal{S}_{V}$ and consider the perturbed Jacobi-operator $A_{x}$ defined in (3) as an unbounded operator in $L^{2}=L^{2}\left(S^{1}, x^{*} T M\right)$ with dense domain $W^{2,2}=W^{2,2}\left(S^{1}, x^{*} T M\right)$. It is selfadjoint since it consists of the operator $\frac{d^{2}}{d t^{2}}$ on $S^{1}$ plus a bounded operator.

Theorem 2.2. (Morse index theorem) Let $x \in C r i t$, then the Morse index $\operatorname{Ind}(x)$ and the nullity $\operatorname{Null}(x):=\operatorname{dim}$ ker $A_{x}$ are finite.

Proof. Since $M$ is compact there exists a constant $C>0$ such that

$$
\begin{aligned}
\left\langle\xi, A_{x} \xi\right\rangle_{L^{2}} & =\left\|\nabla_{t} \xi\right\|_{2}^{2}-\langle\xi, R(\xi, \dot{x}) \dot{x}\rangle_{L^{2}}-\left\langle\xi, \nabla_{\xi} \nabla V_{t}(x)\right\rangle_{L^{2}} \\
& \geq\left\|\nabla_{t} \xi\right\|_{2}^{2}-C\|\xi\|_{2}^{2}
\end{aligned}
$$

for all $\xi \in C^{\infty}\left(S^{1}, x^{*} T M\right)$. For any $\rho>C$ it follows that the unbounded operator $A_{x}+\rho$ in $L^{2}$ with dense domain $W^{2,2}$ is positive definite and so in particular injective. Moreover, it is selfadjoint and therefore also surjective with real spectrum. When viewed as a bounded operator from $W^{2,2}$ to $L^{2}$ the open mapping theorem guarantees existence of a bounded inverse. Together with a standard compact Sobolev embedding we obtain that the resolvent operator is compact:

$$
\left(A_{x}+\rho\right)^{-1}: L^{2}\left(S^{1}, x^{*} T M\right) \stackrel{\text { bd. }}{\longrightarrow} W^{2,2}\left(S^{1}, x^{*} T M\right) \stackrel{\text { cp. }}{\hookrightarrow} L^{2}\left(S^{1}, x^{*} T M\right)
$$

Compactness implies discrete spectrum, say $\left\{1 / \mu_{j}\right\}_{j \in \mathbb{N}}$, with finite multiplicities and possible accumulation point at 0 . We observe that $1 / \mu_{j}$ eigenvalue of $\left(A_{x}+\rho\right)^{-1}$ if and only if $\mu_{j}$ is an eigenvalue of $A_{x}+\rho$. We already know that $\mu_{j}>0$ and conclude $\mu_{j} \rightarrow+\infty$ for $j \rightarrow \infty$. Clearly,

$$
\left(A_{x}+\rho\right)^{-1} \xi_{j}=\frac{1}{\mu_{j}} \xi_{j} \quad \Longleftrightarrow \quad A_{x} \xi_{j}=\left(\mu_{j}-\rho\right) \xi_{j}
$$

Hence the eigenvalues $\left(\mu_{j}-\rho\right)$ of $A_{x}$ tend to $+\infty$ for $j \rightarrow \infty$ which proves the Morse index theorem.

2.4. Conley-Zehnder index. Let us first illustrate the Conley-Zehnder index $\mu_{C Z}$ for a certain class of paths in $S p(2 n)$, introduced in 1984 by Conley and Zehnder CZ84. Later on we shall give a precise definition of the more general Robbin-Salamon index $\mu_{R S}$ which will be more convenient to carry out calculations.

Let $S p^{ \pm}(2 n)=\{Y \in S p(2 n) \mid \operatorname{det}(\mathbb{1}-Y) \gtrless 0\}, S p^{*}(2 n)=S p^{+}(2 n) \cup$ $S p^{-}(2 n), \mathcal{C}(2 n)=S p(2 n) \backslash S p^{*}(2 n)$ and $\mathcal{S P}(2 n)$ be the set of admissible paths, which by definition means continous paths $\gamma:[0,1] \rightarrow S p(2 n)$ such that $\gamma(0)=\mathbb{1}$ and $\gamma(1) \in S p^{*}(2 n)$. The Maslov cycle $\mathcal{C}(2 n)$ is a codimension one algebraic subvariety of $S p(2 n)$ and it is possible to interpret $\mu_{C Z}(\gamma)$ as the algebraic intersection number of a generic path $\gamma \in \mathcal{S P}(2 n)$ with the Maslov cycle $\mathcal{C}(2 n)$. Generic means that $\gamma$ is of class $C^{1}$ and that the 
intersection of $\gamma$ with $\mathcal{C}(2 n)$ is transversal for $t>0$. Moreover, we need to assume that $\gamma$ departs from $\mathbb{1}$ at $t=0$ into $S p^{-}(2 n)$; otherwise we homotop $\gamma$ within $\mathcal{S P}(2 n)$ to another path $\gamma^{\prime}$ satisfying the additional condition and define $\mu_{C Z}(\gamma)=\mu_{C Z}\left(\gamma^{\prime}\right)$.

For $n=1$ one can identify the symplectic linear group with the interior of the solid 2-torus GL58; in this case $\mathcal{C}(2)$ has precisely one singularity which corresponds to the identity matrix. Figure 1 (a) shows a numerical plot of the path $\gamma_{1}:[0,1] \rightarrow S p(2)$

$$
\gamma_{1}(t)=\left(\begin{array}{cc}
\cos \pi t & -\sin \pi t \\
\sin \pi t & \cos \pi t
\end{array}\right)\left(\begin{array}{cc}
1+t & 0 \\
0 & (1+t)^{-1}
\end{array}\right)
$$

which has Conley-Zehnder index +1 . In (b) the path $\gamma_{2}$ from (32) is shown and it is important to notice that it departs from $\mathbb{1}$ at $t=0$ not into $\mathrm{Sp}^{-}(2)$, but into $S p^{+}(2)$. However, the path $\gamma_{2}$ is homotopic within $\mathcal{S P}(2)$ to $\gamma_{1}$ and therefore its Conley-Zehnder index is also +1 .

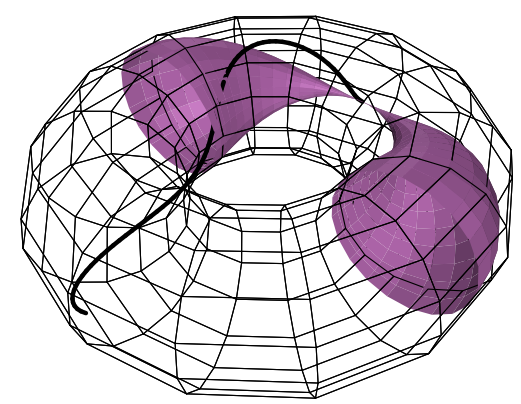

(a) The path $\gamma_{1}$

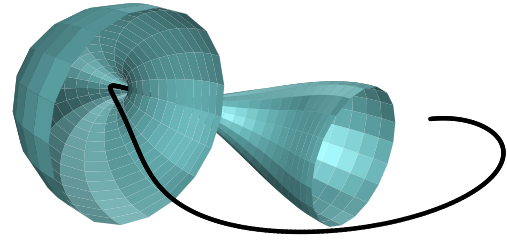

(b) The path $\gamma_{2}$

Figure 1. Maslov cycle $\mathcal{C}(2)$ and paths with $\mu_{C Z}=+1$

We observed in section 2.1 that for any $x \in C$ rit we obtain a 1-periodic orbit of the Hamiltonian system $\left(T^{*} M, \omega_{c a n}, H=H_{V}\right)$ by setting $z=z_{x}=$ $\left(x, g(x) \partial_{t} x\right)$ and every 1-periodic orbit is obtained this way. Let $\varphi_{t}: T^{*} M \rightarrow$ $T^{*} M$ denote the time-t-map generated by the Hamiltonian vector field $X_{H}$, which is defined by $\omega_{\text {can }}\left(X_{H}, \cdot\right)=d H(\cdot)$, let $z_{0}=z(0)$ and $\Phi_{U}$ be the unitary trivialization of $x^{*} T M \oplus x^{*} T^{*} M \rightarrow S^{1}$ introduced in (12). Then we obtain a smooth path of symplectic matrices by linearizing the flow along the orbit $z(t)=\varphi_{t} z_{0}$

$$
\gamma(t)=\gamma_{x, U, \phi}(t):=\Phi_{U}(t)^{-1} d \varphi_{t}\left(z_{0}\right) \Phi_{U}(0)
$$

Clearly $\gamma(0)=\mathbb{1}$ and the second condition $\gamma(1) \in S p^{*}$ is equivalent to ker $A_{x}=\{0\}$, which reflects our choice $V \in \mathcal{V}_{\text {reg }}$. 
Definition 2.3. For $x \in C$ rit such that $\operatorname{ker} A_{x}=\{0\}$ we define the ConleyZehnder index of the 1-periodic orbit $z_{x}=g(x) \partial_{t} x$ by

$$
\mu_{C Z}\left(z_{x}\right):=\mu_{C Z}\left(\gamma_{x, U, \phi}\right)
$$

where $\gamma_{x, U, \phi}$ is as in (14).

The following lemma shows that this definition is actually independent of the choice of $\phi$. Its proof will be given in subsection 2.5.

Lemma 2.4. (i) $\mu_{C Z}\left(\gamma_{x, U, \phi}\right)=\mu_{C Z}\left(\gamma_{x, U, \tilde{\phi}}\right)$ for any $\phi, \tilde{\phi}$ as in (\$). (ii) $\mu_{C Z}\left(\gamma_{x, U^{2 k+1}}\right)=2 k \sigma(x)+\mu_{C Z}\left(\gamma_{x, U}\right)$ for any $k \in \mathbb{Z}$.

Remark 2.5. (1) In the standard setting of Floer homology, namely that of a closed symplectic manifold $(N, \omega)$, one only considers contractible 1periodic orbits $z$ and trivializes $z^{*} T M \rightarrow S^{1}$ by filling in a disk. However, the Conley-Zehnder index of the resulting path in $S p(2 n)$ might depend on the homotopy class of the disk. An ambiguity arises precisely in the presence of spheres $\iota: S^{2} \hookrightarrow N$ with nonvanishing first Chern class $c_{1}\left(\iota^{*} T N\right)$; cf. the exposition Sa99]. Here this cannot happen, since $c_{1}\left(\iota^{*} T W\right)=0$ for any closed submanifold $\iota: W \hookrightarrow T^{*} M$; e.g. see [We99, section B.1.7] for two different proofs.

(2) The reason that in the present case the Conley-Zehnder index is well defined for any closed orbit $x$ with $\sigma(x)=0$, contractible or not, is the existence of the global Lagrangian splitting $T_{z} T^{*} M \simeq T_{x} M \oplus T_{x}^{*} M, z \in$ $T_{x}^{*} M$. However, as mentioned earlier, a $(\bmod 2)$-ambiguity arises in case of nontrivial bundles $x^{*} T M \rightarrow S^{1}$.

In order to prove the index theorem it will be useful to represent the path $\gamma$ in (14) as solution $\Psi=\Psi_{U}$ of the initial value problem stated in the following lemma.

Lemma 2.6. The path of symplectic matrices $\gamma$ defined in (14) equals the fundamental solution $\Psi$ of the initial value problem

$$
\partial_{t} \Psi=-J_{0} S_{U} \Psi, \quad \Psi(0)=\mathbb{1}
$$

where $S_{U}$ is the family of symmetric matrices from (13).

Proof. To show equality of $\gamma$ and $\Psi$ we choose the following smooth variation of $z(t)=\varphi_{t}\left(z_{0}\right)$

$$
z^{\tau}=\varphi_{t}\left(\exp _{x_{0}} \tau \xi_{0}, y_{0}+\tau \eta_{0}\right), \quad\left(\xi_{0}, \eta_{0}\right) \in T_{x_{0}} M \oplus T_{x_{0}}^{*} M
$$

in order to linearize (6) as described in subsection 2.1. With this choice $z^{0}(t)=\varphi_{t}\left(z_{0}\right)=z(t)=(x(t), y(t))$ and

$$
\left(\begin{array}{l}
\xi(t) \\
\eta(t)
\end{array}\right):=\left.\frac{d}{d \tau}\right|_{\tau=0} z^{\tau}(t)=d \varphi_{t}\left(z_{0}\right)\left(\begin{array}{l}
\xi_{0} \\
\eta_{0}
\end{array}\right) \in C^{\infty}\left([0,1], x^{*} T M \oplus x^{*} T^{*} M\right)
$$

is a zero of the linear equations $(\overline{7})$, which however might not close up at time $t=1$. In other words the linearized flow along a flow line (zero of 
the nonlinear equations) provides a zero of the linearized equations. With respect to the unitary frame $\Phi_{U}$ these are given by

$$
0=\left(J_{0} \partial_{t}-S_{U}\right) \Phi_{U}^{-1}\left(\begin{array}{l}
\xi \\
\eta
\end{array}\right)=\left(J_{0} \partial_{t}-S_{U}\right) \gamma
$$

and this proves the claim.

Remark 2.7. Since the flow applied to a point produces a path, the natural domain for the linearized equations along such a path actually is the space of vector fields along the path. If we restrict our attention to those paths which happen to be 1-periodic, then the natural domain becomes the set of vector fields along loops and we obtain the operator $A_{z_{x}}$ from (7).

To summarize, we have that any $(\xi, \eta)$ in the kernel of the linearized flow along a flow line $z=(x, y)$ is of the form $d \varphi_{t}(z(0))\left(\xi_{0}, \eta_{0}\right)$ where $\left(\xi_{0}, \eta_{0}\right) \in$ $T_{x(0)} M \oplus T_{x(0)}^{*} M$. In the periodic case the vector fields are required to close up at time $t=1$ and so the corresponding kernel is isomorphic to a subspace, namely

$$
\operatorname{ker} A_{z_{x}} \simeq \operatorname{ker}\left(\mathbb{1}-d \varphi_{1}(z(0))\right)
$$

2.5. Robbin-Salamon index. In order to prove the index theorem 1.2 as well as lemma 2.4 we shall briefly recall another Maslov-type index $\mu_{R S}$, called Robbin-Salamon index [RS93]. It is defined for any continuous path of Lagrangian subspaces $\Lambda:[a, b] \ni t \mapsto \Lambda(t)$ of a given symplectic vector space $(V, \Omega)$ with respect to a fixed Lagrangian subspace $\Lambda_{0}$.

For now let us assume the path $\Lambda$ is of class $C^{1}$. Call $t_{i} \in[a, b]$ a crossing if $\Lambda\left(t_{i}\right) \cap \Lambda_{0} \neq\{0\}$. For any such $t_{i}$ there is a quadratic form on $\Lambda\left(t_{i}\right)$ : Pick any Lagrangian complement $W$ of $\Lambda\left(t_{i}\right)$ and for $v \in \Lambda\left(t_{i}\right)$ and sufficiently small $\epsilon>0$ define $w(\epsilon) \in W$ by the condition $v+w(\epsilon) \in \Lambda\left(t_{i}+\epsilon\right)$. Then

$$
\hat{Q}\left(\Lambda\left(t_{i}\right), \partial_{t} \Lambda\left(t_{i}\right)\right) v:=\left.\frac{d}{d \epsilon}\right|_{\epsilon=0} \Omega(v, w(\epsilon))
$$

is a quadratic form on $\Lambda\left(t_{i}\right)$, which is independent of the choice of $W$ RS93, theorem 1.1]. The crossing form at $t_{i}$ is its restriction to $\Lambda\left(t_{i}\right) \cap \Lambda_{0}$

$$
\Gamma\left(\Lambda, \Lambda_{0}, t_{i}\right):=\left.\hat{Q}\left(\Lambda\left(t_{i}\right), \partial_{t} \Lambda\left(t_{i}\right)\right)\right|_{\Lambda\left(t_{i}\right) \cap \Lambda_{0}}
$$

and $t_{i}$ is called a regular crossing if its crossing form is nonsingular. A path $\Lambda$ is called regular if all its crossings are regular.

Definition 2.8. The Robbin-Salamon index of a regular Lagrangian path $\Lambda$ is defined to be

$$
\mu_{R S}\left(\Lambda, \Lambda_{0}\right)=\frac{1}{2} \operatorname{sign} \Gamma\left(\Lambda, \Lambda_{0}, a\right)+\sum_{a<t<b} \operatorname{sign} \Gamma\left(\Lambda, \Lambda_{0}, t\right)+\frac{1}{2} \operatorname{sign} \Gamma\left(\Lambda, \Lambda_{0}, b\right)
$$

where the sum runs over all crossings $t$.

We remark that in RS93, section 2] the following is shown: Any two regular Lagrangian paths which are homotopic with fixed endpoints have the same Robbin-Salamon index and every continuous Lagrangian path is 
homotopic with fixed endpoints to a regular one. As a consequence one can define the Robbin-Salamon index for every continuous path.

The most important property of $\mu_{R S}$ in this text will be its catenation property, which means that $\mu_{R S}$ is additive under composition of paths (with respect to decompositions of the parameter domain $[a, b]$ ). Moreover, for any path of symplectic matrices $\Psi \in \mathcal{S P}(2 n)$ the Robbin-Salamon index reproduces the Conley-Zehnder index [RS93, remark 5.3] as follows

$$
\mu_{R S}(\operatorname{Graph} \Psi, \Delta)=\mu_{C Z}(\Psi)
$$

Here $\Delta$ denotes the diagonal in the symplectic vector space $\left(\mathbb{R}^{2 n \times 2 n},-\omega_{0} \oplus\right.$ $\left.\omega_{0}\right)$ and $\omega_{0}(\cdot, \cdot)=\left\langle J_{0}, \cdot \cdot\right\rangle$ is the standard symplectic form on $\mathbb{R}^{2 n}$. The loop property of the Conley-Zehnder index [DS94] translates into

$$
\mu_{R S}(\operatorname{Graph} \Theta \Psi, \Delta)=\mu_{R S}(\operatorname{Graph} \Theta, \Delta)+\mu_{R S}(\operatorname{Graph} \Psi, \Delta)
$$

for any path $\Psi \in \mathcal{S P}(2 n)$ and any loop $\Theta: S^{1} \rightarrow S p(2 n)$.

Lemma 2.9. For every unitary loop

$$
\Theta=\left(\begin{array}{cc}
X & -Y \\
Y & X
\end{array}\right): S^{1} \rightarrow S p(2 n) \cap O(2 n)
$$

it holds

$$
\mu_{R S}(\operatorname{Graph} \Theta, \Delta)=2 \operatorname{deg}\left[\operatorname{det}(X+i Y): S^{1} \rightarrow S^{1}\right]
$$

We only sketch a proof: The idea is to shows that the right hand side satisfies those axioms which determine the left hand side uniquely. These are the direct sum, the normalization and the weak homotopy axioms.

Finally let us derive a formula for the crossing form in case $\Lambda(t)=$ Graph $\Psi(t) \subset\left(\mathbb{R}^{2 n \times 2 n},-\omega_{0} \oplus \omega_{0}\right), \Lambda_{0}=\Delta$ and $\Psi:[a, b] \rightarrow S p(2 n)$ of class $C^{1}$. First observe that $\Psi$ determines a path of symmetric matrices by

$$
S(t)=J_{0}\left(\partial_{t} \Psi(t)\right) \Psi(t)^{-1}
$$

Let $t_{i} \in[a, b]$ be a crossing, $v \in G r a p h \Psi\left(t_{i}\right) \cap \Delta$ and $W=0 \times \mathbb{R}^{2 n}$. Then $v=\left(\zeta, \Psi\left(t_{i}\right) \zeta\right)=(\zeta, \zeta)$ for some $\zeta \in \mathbb{R}^{2 n}, w(\epsilon)=\left(0, w_{2}(\epsilon)\right)$ and the condition $v+w(\epsilon) \in \operatorname{Graph} \Psi\left(t_{i}+\epsilon\right)$ leads to $w_{2}(\epsilon)=\Psi\left(t_{i}+\epsilon\right) \zeta-\zeta$. We calculate

$$
\begin{aligned}
\hat{Q}\left(\Lambda\left(t_{i}\right), \partial_{t} \Lambda\left(t_{i}\right)\right) v & =\left.\frac{d}{d \epsilon}\right|_{\epsilon=0}\left(-\omega_{0} \oplus \omega_{0}\right)\left((\zeta, \zeta),\left(0, w_{2}(\epsilon)\right)\right) \\
& =\left.\frac{d}{d \epsilon}\right|_{\epsilon=0}\left(-\omega_{0}(\zeta, 0)+\omega_{0}\left(\zeta, \Psi\left(t_{i}+\epsilon\right) \zeta-\zeta\right)\right) \\
& =\omega_{0}\left(\zeta, \partial_{t} \Psi\left(t_{i}\right) \zeta\right) \\
& =-\left\langle\zeta, S\left(t_{i}\right) \Psi\left(t_{i}\right) \zeta\right\rangle \\
& =-\left\langle\zeta, S\left(t_{i}\right) \zeta\right\rangle
\end{aligned}
$$

and, identifying $\left(\operatorname{Graph} \Psi\left(t_{i}\right)\right) \cap \Delta$ and $\operatorname{ker}\left(\mathbb{1}-\Psi\left(t_{i}\right)\right)$ by $(\zeta, \zeta) \mapsto \zeta$, we obtain

$$
\Gamma\left(\operatorname{Graph} \Psi, \Delta, t_{i}\right)(\zeta, \zeta)=-\left\langle\zeta, S\left(t_{i}\right) \zeta\right\rangle, \quad \zeta \in \operatorname{ker}\left(\mathbb{1}-\Psi\left(t_{i}\right)\right)
$$

Now we are in position to prove lemma 2.4. 
Proof. (of Lemma 2.4) ad (i) Consider the transition maps $\psi=\phi^{-1} \tilde{\phi}$ : $[0,1] \rightarrow O(n)$ and $\Psi=\Phi^{-1} \tilde{\Phi}:[0,1] \rightarrow S p(2 n) \cap O(2 n)$, then

$$
\begin{aligned}
& \mu_{C Z}\left(\gamma_{x, U, \phi}\right)=\mu_{C Z}\left(U^{\sigma} \Psi U^{-\sigma} \gamma_{x, U, \tilde{\phi}} U(0)^{\sigma} \Psi(0)^{-1} U(0)^{-\sigma}\right) \\
& \quad=\mu_{R S}\left(\operatorname{Graph} U^{\sigma} \Psi U^{-\sigma}, \Delta\right)+\mu_{C Z}\left(\gamma_{x, U, \tilde{\phi}}\right)+\mu_{R S}\left(\operatorname{Graph} \Psi(0)^{-1}, \Delta\right) \\
& \quad=\mu_{C Z}\left(\gamma_{x, U, \tilde{\phi}}\right)
\end{aligned}
$$

where in the second equality we used (15) as well as the loop property (16). Since $\Psi(0)^{-1}$ is a constant loop the corresponding term vanishes. It remains to show $\mu_{R S}\left(\operatorname{Graph} U^{\sigma} \Psi U^{-\sigma}, \Delta\right)=0$ : Since $U^{\sigma} \Psi U^{-\sigma}:[0,1] \rightarrow$ $S p(2 n) \cap O(2 n)$ we can write

$$
U^{\sigma} \Psi U^{-\sigma}=\left(\begin{array}{cc}
X & -Y \\
Y & X
\end{array}\right) \text { where } X+i Y \in U(n)
$$

and by lemma 2.9 the following is true:

$$
\mu_{R S}\left(\operatorname{Graph} U^{\sigma} \Psi U^{-\sigma}, \Delta\right)=2 \operatorname{deg}\left[\operatorname{det}(X+i Y): S^{1} \rightarrow S^{1}\right]
$$

Now in case $\sigma=0$ we have $X=\psi, Y=0$ and are done. If $\sigma=1$ a calculation shows that

$$
\operatorname{det}(X+i Y)=\operatorname{det}\left(\begin{array}{cccc}
\psi_{11} & e^{-i \pi t} \psi_{12} & \ldots & e^{-i \pi t} \psi_{1 n} \\
e^{i \pi t} \psi_{21} & \psi_{22} & \ldots & \psi_{2 n} \\
\vdots & \vdots & & \vdots \\
e^{i \pi t} \psi_{n 1} & \psi_{n 2} & \ldots & \psi_{n n}
\end{array}\right)=\operatorname{det} \psi= \pm 1
$$

ad (ii)

$$
\begin{aligned}
\mu_{C Z} & \left(\gamma_{x, U^{2 k+1}}\right)=\mu_{C Z}\left(U^{2 k \sigma} \gamma_{x, U} U(0)^{-2 k \sigma}\right) \\
& =\mu_{R S}\left(\operatorname{Graph} U^{2 k \sigma}, \Delta\right)+\mu_{C Z}\left(\gamma_{x, U}\right)+\mu_{R S}\left(\operatorname{Graph} U(0)^{-2 k \sigma}, \Delta\right) \\
& =2 \sigma \operatorname{deg}\left(t \mapsto e^{\pi i 2 k t}\right)+\mu_{C Z}\left(\gamma_{x, U}\right) \\
& =2 k \sigma+\mu_{C Z}\left(\gamma_{x, U}\right)
\end{aligned}
$$

where we used (15) as well as the loop property (16) in the second equality. The third one follows from lemma 2.9.

2.6. Spectral flow and Conley Zehnder index. We provide the main tool to prove the index theorem, namely theorem 2.10 below, which relates the spectral flow and the Conley-Zehnder index.

Pick $T>0$ and smooth two-parameter families of matrices $Q, P$ : $[-T, T] \times[0,1] \rightarrow \mathbb{R}^{n \times n}$ such that

$$
Q_{\lambda}(t)^{T}=Q_{\lambda}(t), \quad Q_{\lambda}(1)=E_{\sigma} Q_{\lambda}(0) E_{\sigma}^{-1}
$$

and

$$
P_{\lambda}(t)^{T}=-P_{\lambda}(t), \quad P_{\lambda}(1)=E_{\sigma} P_{\lambda}(0) E_{\sigma}^{-1}
$$


For $\lambda \in[-T, T]$ consider the family $A_{\lambda}$ of selfadjoint operators in $L_{\sigma}^{2}$ with dense domain $W_{\sigma}^{2,2}$ defined by

$$
A_{\lambda}(\xi):=-\partial_{t} \partial_{t} \xi-B_{\lambda} \xi-Q_{\lambda} \xi
$$

where $L_{\sigma}^{2}$ and $W_{\sigma}^{2,2}$ have been introduced in section 2.3 and the $L^{2}$ symmetric family of first order operators $B_{\lambda}$ is defined by

$$
B_{\lambda} \xi=2 P_{\lambda} \dot{\xi}+\dot{P}_{\lambda} \xi+P_{\lambda}^{2} \xi
$$

The term $\partial_{t} \partial_{t}+B_{\lambda}$ represents the second covariant derivative $\nabla_{t} \nabla_{t}$ with respect to an orthonormal frame. To indicate the particular choices $\lambda=\mp T$ we frequently will use the simpler notation $\mp$.

Let us now define the upward spectral flow of the family $A_{\lambda}, \lambda \in[-T, T]$. Roughly speaking, it counts the number of eigenvalues changing sign from minus to plus during the deformation minus the ones changing sign in the opposite way. The real number $\lambda$ is called a crossing, if $A_{\lambda}$ is not injective. In this case, following the exposition in RS95], we define the crossing operator

$$
\Gamma\left(\left\{A_{\lambda}\right\}_{\lambda \in[-T, T]}, \lambda\right)=\left.P_{\lambda}^{\perp}\left(\partial_{\lambda} A_{\lambda}\right) P_{\lambda}^{\perp}\right|_{K e r} A_{\lambda}
$$

where $P_{\lambda}^{\perp}: L_{\sigma}^{2} \rightarrow L_{\sigma}^{2}$ denotes the orthogonal projection onto $\operatorname{Ker} A_{\lambda}$. We emphasize that, despite the similarity of notation, the object $P_{\lambda}^{\perp}$ is entirely different from $P_{\lambda}$ and that sign below denotes the signature of a quadratic form. A crossing is called regular, if its crossing operator is nonsingular. The spectral flow is characterized axiomatically in [RS95]. In case all crossings are regular we may use lemma 4.27 in RS95 to actually define

$$
\mu_{\text {Spec }}\left(\left\{A_{\lambda}\right\}_{\lambda \in[-T, T]}\right)=\sum_{\lambda} \operatorname{sign} \Gamma\left(\left\{A_{\lambda}\right\}_{\lambda \in[-T, T]}, \lambda\right)
$$

Note that the sum is over all crossings $\lambda$ and that there are only finitely many of them in view of their regularity.

In what follows we need to assume injectivity of $A_{\mp}$ :

$$
\text { assume that } \operatorname{Ker} A_{\mp}=\{0\}
$$

Let us now state the main theorem of this section.

Theorem 2.10. Let $\left\{A_{\lambda}\right\}_{\lambda \in[-T, T]}$ be a regular family as in (19) and assume injectivity of $A_{\mp}$. Let $\Psi_{\mp, U}$ be the symplectic paths associated to $A_{\mp}$ by the equivalence of statements (S1) and (S4) below, then the upward spectral flow of the family is given by

$$
\mu_{\text {Spec }}\left(\left\{A_{\lambda}\right\}_{\lambda \in[-T, T]}\right)=\mu_{C Z}\left(\Psi_{+, U}\right)-\mu_{C Z}\left(\Psi_{-, U}\right)
$$

Equivalence of the subsequent four statements is fairly easy, but nevertheless crucial in the proof of the theorem. Together they show how the operator $A_{\lambda}$ leads to a path $\Psi_{\lambda, U}$ of symplectic matrices. Throughout let $\xi \in C^{\infty}\left([0,1], \mathbb{R}^{n}\right)$, denote $\eta=\dot{\xi}+P_{\lambda} \xi$ and

$$
S_{\lambda}=S_{\lambda}(t)=\left(\begin{array}{cc}
Q_{\lambda} & P_{\lambda} \\
-P_{\lambda} & \mathbb{1}_{n}
\end{array}\right), \quad\left(\begin{array}{c}
\xi_{U} \\
\eta_{U}
\end{array}\right)=U^{\sigma}\left(\begin{array}{c}
\xi \\
\eta
\end{array}\right)
$$


where the rotation $U$ is defined in (11) and $\sigma \in\{0,1\}$.

(S1)

$$
-\partial_{t} \partial_{t} \xi-B_{\lambda} \xi-Q_{\lambda} \xi=0
$$

$$
\partial_{t}\left(\begin{array}{l}
\xi \\
\eta
\end{array}\right)=-J_{0} S_{\lambda}\left(\begin{array}{l}
\xi \\
\eta
\end{array}\right)
$$

$$
\left(\begin{array}{l}
\xi(t) \\
\eta(t)
\end{array}\right)=\Psi_{\lambda}(t)\left(\begin{array}{l}
\xi(0) \\
\eta(0)
\end{array}\right)
$$

and the fundamental solution $\Psi_{\lambda}(t)$ is determined by

$$
\partial_{t} \Psi_{\lambda}=-J_{0} S_{\lambda} \Psi_{\lambda}, \quad \Psi_{\lambda}(0)=\mathbb{1}_{2 n}
$$

$$
\left(\begin{array}{l}
\xi_{U} \\
\eta_{U}
\end{array}\right)(t)=\Psi_{\lambda, U}(t)\left(\begin{array}{l}
\xi_{U} \\
\eta_{U}
\end{array}\right)(0)
$$

and the fundamental solution $\Psi_{\lambda, U}(t)$ is determined by

$$
\begin{gathered}
\partial_{t} \Psi_{\lambda, U}=-J_{0} S_{\lambda, U} \Psi_{\lambda, U}, \quad \Psi_{\lambda, U}(0)=\mathbb{1}_{2 n} \\
S_{\lambda, U}=U^{\sigma} S_{\lambda} U^{-\sigma}-J_{0} U^{\sigma} \partial_{t}\left(U^{-\sigma}\right)
\end{gathered}
$$

Remark 2.11. If in addition we require $\xi$ in (S1) to satisfy the boundary conditions $\xi(1)=E_{\sigma} \xi(0)$ and $\dot{\xi}(1)=E_{\sigma} \dot{\xi}(0)$, i.e. $\xi \in \operatorname{ker} A_{\lambda}$, it turns out that in $(\mathrm{S} 3)$

$$
\left(\begin{array}{l}
\xi(0) \\
\eta(0)
\end{array}\right) \in \operatorname{ker}\left(E_{\sigma} \oplus E_{\sigma}-\Psi_{\lambda}(1)\right)
$$

and in (S4)

$$
\left(\begin{array}{l}
\xi_{U}(0) \\
\eta_{U}(0)
\end{array}\right) \in \operatorname{ker}\left(\mathbb{1}-\Psi_{\lambda, U}(1)\right)
$$

In view of the injectivity assumption (22) the symplectic paths $\Psi_{\mp, U}$ end outside the Maslov cycle and therefore are elements of the set $\mathcal{S P}(2 n)$ for which the Conley-Zehnder index is defined.

Proof. (of Theorem 2.10) For each $\lambda \in[-T, T]$ there is the path of symplectic matrices $\Psi_{\lambda, U}$ defined in (S4). Together these give rise to a 2-parameter family of Lagrangian subspaces

$$
\Lambda_{\lambda}(t)=\operatorname{Graph} \Psi_{\lambda, U}(t) \subset\left(\mathbb{R}^{2 n} \times \mathbb{R}^{2 n},-\omega_{0} \oplus \omega_{0}\right)
$$

and we consider the Robbin-Salamon index $\mu_{R S}(\Gamma, \Delta)$ of the loop of Lagrangians $\Gamma$ along the boundary of the parameter domain $[0,1] \times[-T, T]$. We may break down the loop $\Gamma$ into the four subpaths $\gamma_{1}, \ldots, \gamma_{4}$ indicated in 


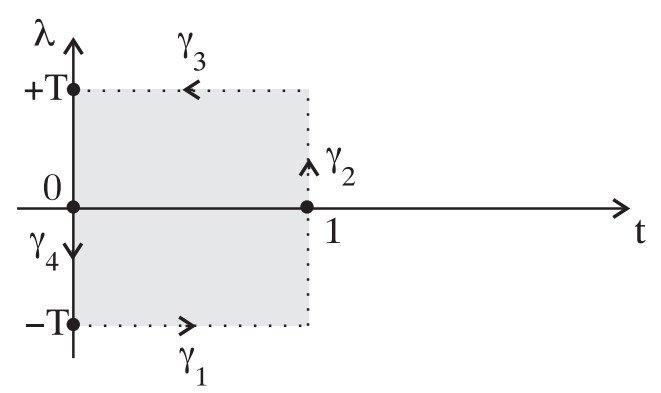

Figure 2. Contractible loop of Lagrangian subspaces

figure 2. $\Gamma$ is contractible and so its Robbin-Salamon index is zero. Together with the catenation property of $\mu_{R S}$ this leads to

$$
0=\mu_{R S}(\Gamma, \Delta)=\sum_{i=1}^{4} \mu_{R S}\left(\gamma_{i}, \Delta\right)
$$

Since $\Psi_{\lambda, U}(0)=\mathbb{1}_{2 n}, \gamma_{4} \equiv \Delta$ and so $\mu_{R S}\left(\gamma_{4}, \Delta\right)=0$. Moreover, as indicated in (15) the Robbin-Salamon index of $\gamma_{1}$ is precisely the Conley-Zehnder index of the corresponding symplectic path. We get a minus sign in the case of $\gamma_{3}$, because of its reversed orientation

$$
\begin{aligned}
& \mu_{R S}\left(\gamma_{1}, \Delta\right)=\mu_{R S}\left(\operatorname{Graph} \Psi_{-, U}, \Delta\right)=\mu_{C Z}\left(\Psi_{-, U}\right) \\
& \mu_{R S}\left(\gamma_{3}, \Delta\right)=-\mu_{R S}\left(\operatorname{Graph} \Psi_{+, U}, \Delta\right)=-\mu_{C Z}\left(\Psi_{+, U}\right)
\end{aligned}
$$

It remains to show

$$
\mu_{R S}\left(\gamma_{2}, \Delta\right)=\mu_{\text {spec }}\left(\left\{A_{\lambda}\right\}_{\lambda \in[-T, T]}\right)
$$

In order to prove (23) we need to derive two identities. Recall from (S4) that $\Psi_{\lambda, U}$ is determined by

$$
\partial_{t} \Psi_{\lambda, U}(t)=-J_{0} S_{\lambda, U}(t) \Psi_{\lambda, U}(t), \quad \Psi_{\lambda, U}(0)=\mathbb{1}
$$

Now for fixed $t \in[0,1]$ the path $\lambda \mapsto \Psi_{\lambda, U}(t), \lambda \in[-T, T]$, leads to another path of symmetric matrices

$$
\hat{S}_{t}(\lambda)=J_{0}\left(\partial_{\lambda} \Psi_{\lambda, U}(t)\right) \Psi_{\lambda, U}(t)^{-1}
$$


Note that $\hat{S}_{0}(\lambda) \equiv 0$, since $\Psi_{\lambda, U}(0) \equiv \mathbb{1}$. Using these equations we obtain

$$
\begin{aligned}
\partial_{t}\left(\Psi_{\lambda, U}(t)^{T}\right. & \left.\hat{S}_{t}(\lambda) \Psi_{\lambda, U}(t)\right) \\
= & \left(\partial_{t} \Psi_{\lambda, U}(t)^{T}\right) \hat{S}_{t}(\lambda) \Psi_{\lambda, U}(t)+\Psi_{\lambda, U}(t)^{T} \partial_{t}\left(J_{0} \partial_{\lambda} \Psi_{\lambda, U}(t)\right) \\
= & \Psi_{\lambda, U}(t)^{T} S_{\lambda, U}(t) J_{0} \hat{S}_{t}(\lambda) \Psi_{\lambda, U}(t) \\
& -\Psi_{\lambda, U}(t)^{T} J_{0} \partial_{\lambda}\left(J_{0} S_{\lambda, U}(t) \Psi_{\lambda, U}(t)\right) \\
= & -\Psi_{\lambda, U}(t)^{T} S_{\lambda, U}(t) \partial_{\lambda} \Psi_{\lambda, U}(t)+\Psi_{\lambda, U}(t)^{T}\left(\partial_{\lambda} S_{\lambda, U}(t)\right) \Psi_{\lambda, U}(t) \\
& +\Psi_{\lambda, U}(t)^{T} S_{\lambda, U}(t) \partial_{\lambda} \Psi_{\lambda, U}(t) \\
= & \Psi_{\lambda, U}(t)^{T}\left(\partial_{\lambda} S_{\lambda, U}(t)\right) \Psi_{\lambda, U}(t)
\end{aligned}
$$

Integration over $t$ from 0 to 1 , together with $\hat{S}_{0}(\lambda) \equiv 0$, leads to the first identity we are looking for, namely

$$
\Psi_{\lambda, U}(1)^{T} \hat{S}_{1}(\lambda) \Psi_{\lambda, U}(1)=\int_{0}^{1} \Psi_{\lambda, U}(t)^{T}\left(\partial_{\lambda} S_{\lambda, U}(t)\right) \Psi_{\lambda, U}(t) d t
$$

Use skew-symmetry of $P_{\lambda}$ and $\partial_{\lambda} P_{\lambda}$ as well as $\eta=\dot{\xi}+P_{\lambda} \xi$ and

$$
\partial_{\lambda} B_{\lambda}=2\left(\partial_{\lambda} P_{\lambda}\right) \partial_{t}+\partial_{\lambda} \dot{P}_{\lambda}+\left(\partial_{\lambda} P_{\lambda}\right) P_{\lambda}+P_{\lambda}\left(\partial_{\lambda} P_{\lambda}\right)
$$

to obtain the second identity

$$
\begin{aligned}
\int_{0}^{1}\left\langle\left(\begin{array}{l}
\xi \\
\eta
\end{array}\right),\left(\begin{array}{cc}
-\partial_{\lambda} B_{\lambda} & \partial_{\lambda} P_{\lambda} \\
-\partial_{\lambda} P_{\lambda} & 0
\end{array}\right)\left(\begin{array}{l}
\xi \\
\eta
\end{array}\right)\right\rangle_{\mathbb{R}^{2 n}} d t \\
=\int_{0}^{1}\left\langle\xi,-2\left(\partial_{\lambda} P_{\lambda}\right) \dot{\xi}-\left(\partial_{\lambda} \dot{P}_{\lambda}\right) \xi-P_{\lambda}\left(\partial_{\lambda} P_{\lambda}\right) \xi-\left(\partial_{\lambda} P_{\lambda}\right) P_{\lambda} \xi\right\rangle_{\mathbb{R}^{2 n}} \\
\quad-2\left\langle\dot{\xi}+P_{\lambda} \xi,\left(\partial_{\lambda} P_{\lambda}\right) \xi\right\rangle_{\mathbb{R}^{2 n}} d t \\
=\int_{0}^{1}\left\langle\xi,-\left(\partial_{\lambda} \dot{P}_{\lambda}\right) \xi-P_{\lambda}\left(\partial_{\lambda} P_{\lambda}\right) \xi+\left(\partial_{\lambda} P_{\lambda}\right) P_{\lambda} \xi\right\rangle_{\mathbb{R}^{2 n}} d t \\
=-\int_{0}^{1}\left\langle\xi,\left(\partial_{t} \partial_{\lambda} P_{\lambda}\right) \xi\right\rangle_{\mathbb{R}^{2 n}} d t \\
=-\int_{0}^{1}\left\langle\dot{\xi},\left(\partial_{\lambda} P_{\lambda}\right) \xi\right\rangle_{\mathbb{R}^{2 n}} d t-\int_{0}^{1}\left\langle\xi,\left(\partial_{t} \partial_{\lambda} P_{\lambda}\right) \xi+\left(\partial_{\lambda} P_{\lambda}\right) \dot{\xi}\right\rangle_{\mathbb{R}^{2 n}} d t \\
=-\int_{0}^{1} \partial_{t}\left\langle\xi,\left(\partial_{\lambda} P_{\lambda}\right) \xi\right\rangle_{\mathbb{R}^{2 n}} d t \\
=-\left\langle\xi(1),\left(\partial_{\lambda} P_{\lambda}\right)(1) \xi(1)\right\rangle_{\mathbb{R}^{2 n}}+\left\langle\xi(0),\left(\partial_{\lambda} P_{\lambda}\right)(0) \xi(0)\right\rangle_{\mathbb{R}^{2 n}}=0
\end{aligned}
$$

where in the last equality we used the boundary conditions for $\xi$ and the skewsymmetric family of matrices $P_{\lambda}$. We apply both identities to derive one more crucial result. Let $\xi \in \operatorname{Ker} A_{\lambda}$ and use the symmetry of the projection 
operator $P_{\lambda}^{\perp}$ to obtain

$$
\begin{aligned}
& \left\langle\xi, P_{\lambda}^{\perp}\left(\partial_{\lambda} A_{\lambda}\right) P_{\lambda}^{\perp} \xi\right\rangle_{L^{2}} \\
& =\left\langle\xi,\left(\partial_{\lambda} A_{\lambda}\right) \xi\right\rangle_{L^{2}}=\left\langle\xi,\left(-\partial_{\lambda} B_{\lambda}-\partial_{\lambda} Q_{\lambda}\right) \xi\right\rangle_{L^{2}} \\
& =\int_{0}^{1}\left\langle\left(\begin{array}{l}
\xi \\
\eta
\end{array}\right),\left(\begin{array}{cc}
-\partial_{\lambda} B_{\lambda}-\partial_{\lambda} Q_{\lambda} & 0 \\
0 & 0
\end{array}\right)\left(\begin{array}{l}
\xi \\
\eta
\end{array}\right)\right\rangle_{\mathbb{R}^{2 n}} d t \\
& =\int_{0}^{1}\left\langle\left(\begin{array}{c}
\xi \\
\eta
\end{array}\right),\left(-U^{-\sigma}\left(\partial_{\lambda} S_{\lambda, U}\right) U^{\sigma}+\left(\begin{array}{cc}
-\partial_{\lambda} B_{\lambda} & \partial_{\lambda} P_{\lambda} \\
-\partial_{\lambda} P_{\lambda} & 0
\end{array}\right)\right)\left(\begin{array}{l}
\xi \\
\eta
\end{array}\right)\right\rangle_{\mathbb{R}^{2 n}} d t \\
& =-\left\langle\left(\begin{array}{l}
\xi(0) \\
\eta(0)
\end{array}\right),\left(\int_{0}^{1} \Psi_{\lambda, U}(t)^{T}\left(\partial_{\lambda} S_{\lambda, U}\right)(t) \Psi_{\lambda, U}(t) d t\right)\left(\begin{array}{l}
\xi(0) \\
\eta(0)
\end{array}\right)\right\rangle_{\mathbb{R}^{2 n}} \\
& =-\left\langle\Psi_{\lambda, U}(1)\left(\begin{array}{l}
\xi(0) \\
\eta(0)
\end{array}\right), \hat{S}_{1}(\lambda) \Psi_{\lambda, U}(1)\left(\begin{array}{l}
\xi(0) \\
\eta(0)
\end{array}\right)\right\rangle_{\mathbb{R}^{2 n}} \\
& =-\left\langle\zeta(0), \hat{S}_{1}(\lambda) \zeta(0)\right\rangle_{\mathbb{R}^{2 n}}
\end{aligned}
$$

where we used (25) as well as orthogonality of $U(t)$ in the fifth equality, the identity (24) in the last but one equality and $\zeta(0)=(\xi(0), \eta(0))=$ $\left(\xi_{U}(0), \eta_{U}(0)\right) \in \operatorname{Ker}\left(\mathbb{1}-\Psi_{\lambda, U}(1)\right)$ in the last one.

We are ready to prove (23). The first two equalities are by definition of the spectral flow and the crossing operator

$$
\begin{aligned}
\mu_{\text {Spec }}\left(\left\{A_{\lambda}\right\}_{\lambda \in[-T, T]}\right) & =\left.\sum_{\left\{\lambda \mid \operatorname{Ker} A_{\lambda} \neq\{0\}\right\}} \operatorname{sign} \Gamma\left(\left\{A_{\lambda}\right\}_{\lambda \in[-T, T]}, \lambda\right)\right|_{\text {ker } A_{\lambda}} \\
= & \left.\sum_{\left\{\lambda \mid \operatorname{Ker} A_{\lambda} \neq\{0\}\right\}} \operatorname{sign}\left\langle\cdot, P_{\lambda}\left(\partial_{\lambda} A_{\lambda}\right) P_{\lambda} \cdot\right\rangle_{L^{2}}\right|_{\text {ker } A_{\lambda}} \\
= & \sum_{\left\{\lambda \mid \operatorname{det}\left(\mathbb{1}-\Psi_{\lambda, U}(1)\right) \neq 0\right\}}-\left.\left.\operatorname{sign}\left\langle\cdot, \hat{S}_{1}(\lambda) \cdot\right\rangle_{\mathbb{R}^{2 n}}\right|_{K \operatorname{Ker}\left(\mathbb{1}-\Psi_{\lambda, U}(1)\right)} \operatorname{sign} \Gamma\left(\operatorname{Graph} \Psi_{\cdot, U}(1), \Delta, \lambda\right)\right|_{\Delta \cap \operatorname{Graph} \Psi_{\lambda, U}(1)} \\
= & \sum_{\left\{\lambda \mid \operatorname{Graph} \Psi_{\lambda, U}(1) \cap \Delta \neq\{0\}\right\}} \\
= & \mu_{R S}\left(\operatorname{Graph}\left(\lambda \mapsto \Psi_{\lambda, U}(1)\right), \Delta\right) \\
= & \mu_{R S}\left(\gamma_{2}, \Delta\right)
\end{aligned}
$$

where the third equality is (26). Here it is important that the sums are over the same set of $\lambda^{\prime} s$. This follows from the equivalence of (S1) and (S4)

$$
\xi \in \operatorname{Ker} A_{\lambda} \Longleftrightarrow\left(\begin{array}{c}
\xi(0) \\
\dot{\xi}(0)+P_{\lambda}(0) \xi(0)
\end{array}\right) \in \operatorname{Ker}\left(\mathbb{1}-\Psi_{\lambda, U}(1)\right)
$$

Equality four has been derived in (17), equality five is by definition of $\mu_{R S}$ and the last one is by definition of $\gamma_{2}$. This completes the proof of theorem 2.10 . 


\subsection{Proof of the index theorem 1.2.}

Proof. Let $x \in C$ rit be a nondegenerate critical point, i.e. $\operatorname{ker} A_{x}=\{0\}$, and denote by $\mu_{1} \leq \mu_{2} \leq \ldots \leq \mu_{\operatorname{Ind}(x)}<0$ the negative eigenvalues of $A_{x}$ counted with multiplicities. Fix a real number $\hat{\mu}<\mu_{1}$. It will be convenient in (33) to assume in addition $\hat{\mu}<-\pi$. Let $\beta:[0,1] \rightarrow[\hat{\mu}, 0]$ be a smooth cut-off function which equals 0 near 0 and $\hat{\mu}$ near 1 and is strictly decreasing elsewhere. For $i=1, \ldots, \operatorname{Ind}(x)$ define $\lambda_{i}$ by $\beta\left(\mu_{i}\right)=\lambda_{i}$. Let $Q$ be the path of symmetric matrices defined in (9) and modify $\beta$, if necessary, such that its value at each $\lambda_{i}$ remains the same but $\beta^{\prime}\left(\lambda_{i}\right) \notin \operatorname{Spec} Q(1)$. This technical condition ensures regularity of crossings.

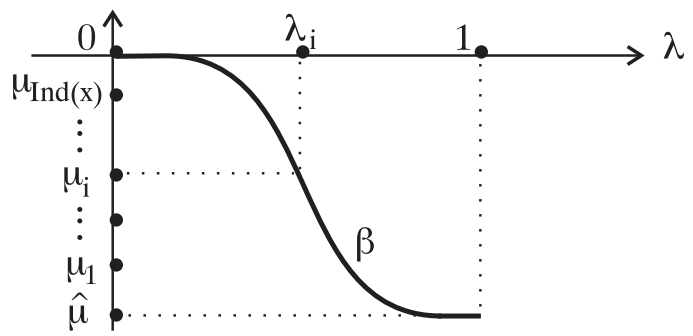

Figure 3. Cut-off function $\beta$ and negative spectrum of $A_{x}$

For $\lambda \in[0,1]$ consider two families of selfadjoint operators in $L_{\sigma}^{2}$ with dense domain $W_{\sigma}^{2,2}$

$$
A_{\lambda} \xi=-\partial_{t} \partial_{t} \xi-B \xi-(1-\lambda) Q \xi-\beta(\lambda) \xi
$$

and

$$
\tilde{A}_{\lambda} \xi=-\partial_{t} \partial_{t} \xi-B_{\lambda} \xi-\hat{\mu} \xi
$$

where

$$
B_{\lambda}=2 P_{\lambda} \partial_{t}+\left(\partial_{t} P_{\lambda}\right)+P_{\lambda}^{2}, \quad P_{\lambda}=(1-\lambda) P
$$

and $B=B_{0}$. The reason to use two families instead of only one is that regularity can be checked easily that way. Both families fit into the framework of section 2.6: Using the boundary conditions for $\xi$ and $\hat{\mu}<0$ a short calculation shows that the second family consists for each $\lambda$ of a positive definite operator and so the injectivity assumption (22) is satisfied. The family is also regular, because there are no crossings at all. To check for the first family we observe that $A_{1}=\tilde{A}_{0}$ is positive definite and $A_{0}$ is precisely the model operator (9) for $A_{x}$. In view of $V \in \mathcal{V}_{\text {reg }}$ this confirms the injectivity assumption (22). Our choice of cut-off function now guarantees regularity of the family. More precisely, assume $\lambda_{i}$ is a crossing and let $\xi \in \operatorname{ker} \partial_{\lambda} A_{\lambda_{i}} \cap \operatorname{ker} A_{\lambda_{i}}$, then the first condition implies

$$
\begin{gathered}
Q(t) \xi(t)=\beta^{\prime}\left(\lambda_{i}\right) \xi(t) \quad \forall t \in[0,1] \\
\xi(0)=E_{\sigma} \xi(1), \dot{\xi}(0)=E_{\sigma} \dot{\xi}(1)
\end{gathered}
$$


Now $\beta^{\prime}\left(\lambda_{i}\right) \notin \operatorname{spec} Q(1)$ implies $\xi(1)=0$. Differentiating (29) with respect to $t$ leads at $t=1$ to

$$
\left(\beta^{\prime}\left(\lambda_{i}\right)-Q(1)\right) \dot{\xi}(1)=\dot{Q}(1) \xi(1)=0
$$

and in view of the regularity of the matrix $\beta^{\prime}\left(\lambda_{i}\right)-Q(1)$ we get $\dot{\xi}(1)=0$. Now we use the second condition which says that $\xi$ is also in the kernel of the second order differential operator $A_{\lambda_{i}}$. Since its boundary conditions are zero, it follows $\xi=0$. This proves nondegeneracy of the crossing operator $\Gamma\left(\left\{A_{\lambda}\right\}_{\lambda \in[0,1]}, \lambda_{i}\right)$ at each crossing $\lambda_{i}$.

We are in position to apply theorem 2.10 to both families. Let us start with the first one and observe that its spectral flow is given by $\operatorname{Ind}\left(A_{0}\right)=$ $\operatorname{Ind}(x)$. We obtain

$$
\operatorname{Ind}(x)=\mu_{C Z}\left(\Psi_{1, U}\right)-\mu_{C Z}\left(\Psi_{0, U}\right)
$$

where according to $(S 4)$ in section 2.6 for each $\lambda \in[0,1]$ the path $\Psi_{\lambda, U}$ : $[0,1] \rightarrow S p(2 n)$ is determined by

$$
\partial_{t} \Psi_{\lambda, U}=-J_{0} S_{\lambda, U} \Psi_{\lambda, U}, \Psi_{\lambda, U}(0)=\mathbb{1}
$$

and

$$
S_{\lambda, U}=U^{\sigma}\left(\begin{array}{cc}
(1-\lambda) Q+\beta(\lambda) \mathbb{1} & P \\
-P & \mathbb{1}
\end{array}\right) U^{-\sigma}-J_{0} U^{\sigma} \partial_{t}\left(U^{-\sigma}\right)
$$

where $\sigma=\sigma(x) \in\{0,1\}$. Observe that $S_{0, U}$ is precisely the matrix $S_{U}$ in lemma (2.6) and so $\Psi_{0, U}$ equals $\gamma$ from (14). In other words $\mu_{C Z}\left(\Psi_{0, U}\right)=$ $\mu_{C Z}\left(z_{x}\right)$.

Since $S_{1, U}$ contains the $t$-dependent matrices $P$ we make use of the second family $\tilde{A}_{\lambda}$ in order to further simplify the problem of calculating $\mu_{C Z}\left(\Psi_{1, U}\right)$. As before we get for each $\lambda \in[0,1]$ a path $\tilde{\Psi}_{\lambda, U}:[0,1] \rightarrow S p(2 n)$ which is determined by

$$
\partial_{t} \tilde{\Psi}_{\lambda, U}=-J_{0} \tilde{S}_{\lambda, U} \tilde{\Psi}_{\lambda, U}, \tilde{\Psi}_{\lambda, U}(0)=\mathbb{1}
$$

where

$$
\tilde{S}_{\lambda, U}=U^{\sigma}\left(\begin{array}{cc}
\hat{\mu} \mathbb{1} & (1-\lambda) P \\
-(1-\lambda) P & \mathbb{1}
\end{array}\right) U^{-\sigma}-J_{0} U^{\sigma} \partial_{t}\left(U^{-\sigma}\right)
$$

The spectral flow of a family of positive definite operators is zero and so theorem 2.10 gives

$$
0=\mu_{C Z}\left(\tilde{\Psi}_{1, U}\right)-\mu_{C Z}\left(\tilde{\Psi}_{0, U}\right)=\mu_{C Z}\left(\tilde{\Psi}_{1, U}\right)-\mu_{C Z}\left(\Psi_{1, U}\right)
$$

where we used $\tilde{S}_{0, U}=S_{1, U}$ in the last step. Hence it remains to show that the Conley-Zehnder index of the path $\tilde{\Psi}_{1, U}$ equals $\sigma(x)$. In order to do so we would like to treat the two cases $\sigma=0$ and $\sigma=1$ separately.

Case 1: $\sigma=0$ We conclude 


$$
\begin{aligned}
& \mu_{C Z}\left(\tilde{\Psi}_{1, U}\right) \\
& =\mu_{R S}\left(\operatorname{Graph} \tilde{\Psi}_{1, U}, \Delta\right) \\
& =\frac{1}{2} \operatorname{sign} \Gamma\left(\operatorname{Graph} \tilde{\Psi}_{1, U}, \Delta, 0\right)=-\left.\frac{1}{2} \operatorname{sign}\left\langle\cdot, \tilde{S}_{1, U}(0) \cdot\right\rangle\right|_{\mathbb{R}^{2 n}} \\
& =-\left.\frac{1}{2} \operatorname{sign}\left\langle\cdot\left(\begin{array}{cc}
\hat{\mu} \mathbb{1} & 0 \\
0 & \mathbb{1}
\end{array}\right) \cdot\right\rangle\right|_{\mathbb{R}^{2 n}} \\
& =0
\end{aligned}
$$

where we used (15) in the first equality, (17) in the third and the fact that there are no further crossings in the second equality.

It remains to show that there are indeed no crossings $t>0$. This is equivalent to 1 not being in the spectrum of $\tilde{\Psi}_{1, U}(t)$ for any $t \in(0,1]$. Because the matrix

$$
\tilde{S}_{1, U}=\left(\begin{array}{cc}
\hat{\mu} \mathbb{1} & 0 \\
0 & \mathbb{1}
\end{array}\right)
$$

is constant in $t$, we can integrate the corresponding differential equation (30) for $\tilde{\Psi}_{1, U}$ and obtain with $\kappa=\sqrt{-\hat{\mu}}$

$$
\tilde{\Psi}_{1, U}(t)=e^{-t J_{0} \tilde{S}_{1, U}}=\exp \left(\begin{array}{cc}
0 & t \mathbb{1} \\
-t \hat{\mu} \mathbb{1} & 0
\end{array}\right)=\left(\begin{array}{cc}
\cosh t \kappa \mathbb{1} & \kappa^{-1} \sinh t \kappa \mathbb{1} \\
\kappa \sinh t \kappa \mathbb{1} & \cosh t \kappa \mathbb{1}
\end{array}\right)
$$

We study the characteristic polynomial of $\tilde{\Psi}_{1, U}(t)$ and obtain its eigenvalues (of multiplicity $n$ each)

$$
\rho_{ \pm}(t)=\cosh t \kappa \pm \sinh t \kappa
$$

Finally the sum (difference) of the hyperbolic cosine and sine is 1 if and only if $t=0$, which shows that there are no further crossings. Let us remark that, because $\tilde{\Psi}_{1, U}(t)$ is symplectic, it follows $\rho_{+}(t)=\rho_{-}(t)^{-1}$ and this is reflected in the key identity for hyperbolic functions $\cosh ^{2}-\sinh ^{2} \equiv 1$.

Case 2: $\sigma=1$ We obtain

$$
\begin{aligned}
& \mu_{C Z}\left(\tilde{\Psi}_{1, U}\right) \\
& =\mu_{R S}\left(\operatorname{Graph} \tilde{\Psi}_{1, U}, \Delta\right) \\
& =\frac{1}{2} \operatorname{sign} \Gamma\left(\operatorname{Graph} \tilde{\Psi}_{1, U}, \Delta, 0\right)=-\left.\frac{1}{2} \operatorname{sign}\left\langle\cdot, \tilde{S}_{1, U}(0) \cdot\right\rangle\right|_{\mathbb{R}^{2 n}} \\
& =-\left.\frac{1}{2} \operatorname{sign}\left\langle\cdot\left(\begin{array}{cc}
\operatorname{diag}_{n}(\hat{\mu}-\pi, \hat{\mu}, \ldots, \hat{\mu}) & 0_{n} \\
0_{n} & \operatorname{diag}_{n}(1-\pi, 1, \ldots, 1)
\end{array}\right) \cdot\right\rangle\right|_{\mathbb{R}^{2 n}} \\
& =1
\end{aligned}
$$

where we used (15) in the first equality, (17) in the third and the fact that there are no further crossings in the second equality: To see this let us rearrange coordinates $\left(x_{1}, \ldots, x_{n}, y_{1}, \ldots, y_{n}\right)$ of $\mathbb{R}^{2 n}$ in the form $\left(x_{1}, y_{1}, x_{2}, \ldots, x_{n}, y_{2}, \ldots, y_{n}\right)$ such that $S p(2 n)$ gets identified with $S p(2) \oplus$ 
$S p(2 n-2)$ and the path of symmetric matrices $\tilde{S}_{1, U}(t)$ from (31) with

$$
\left(\begin{array}{cc}
\hat{\mu} \cos ^{2} \pi t+\sin ^{2} \pi t-\pi & (\hat{\mu}-1) \cos \pi t \sin \pi t \\
(\hat{\mu}-1) \cos \pi t \sin \pi t & \hat{\mu} \sin ^{2} \pi t+\cos ^{2} \pi t-\pi
\end{array}\right) \oplus\left(\begin{array}{cc}
\hat{\mu} \mathbb{1}_{n-1} & 0_{n-1} \\
0_{n-1} & \mathbb{1}_{n-1}
\end{array}\right)
$$

The symplectic path generated by the second term in the direct sum does not meet the Maslov cycle for any $t \in(0,1]$ as was shown in case 1 . Let us denote the first term in the direct sum by $b(t)$. It remains to investigate the path in $S p(2)$ determined by

$$
\partial_{t} \gamma_{2}(t)=-J_{0} b(t) \gamma_{2}(t), \quad \gamma_{2}(0)=\mathbb{1}
$$

and show that there are no crossings with the Maslov cycle $\mathcal{C}$ in $S p(2)$ for any $t \in(0,1]$. A numerical plot of $\gamma_{2}([0,1])$ confirming this in case $\hat{\mu}=-\pi$ is shown in figure 1 (b). To give a proof we introduce the notation

$$
u(t)=\left(\begin{array}{cc}
\cos \pi t & -\sin \pi t \\
\sin \pi t & \cos \pi t
\end{array}\right), \quad s=\left(\begin{array}{cc}
\hat{\mu} & 0 \\
0 & 1
\end{array}\right)
$$

and observe that

$$
b=u s u^{-1}-J_{0} u \partial_{t}\left(u^{-1}\right)
$$

Now $\gamma_{2}$ is a solution of (32) if and only if $\psi=u^{-1} \gamma_{2}$ is a solution of

$$
\partial_{t} \psi=-J_{0} s \psi, \quad \psi(0)=\mathbb{1}
$$

where we used the identity $\pi J_{0}=\left(\partial_{t} u\right) u^{-1}$. The solution $\psi(t)$ is known from case 1 above. We are done once we have shown that the following function is strictly positive for $t \in(0,1]$

$$
\begin{aligned}
f(t) & =\operatorname{det}\left(\mathbb{1}-\gamma_{2}(t)\right)=\operatorname{det}\left(u^{-1}(t)-\psi(t)\right) \\
& =2-2 \cos \pi t \cosh \kappa t+\left(\kappa-\kappa^{-1}\right) \sin \pi t \sinh \kappa t
\end{aligned}
$$

Note that $f(0)=0$ and $f(1)=2(1+\cosh \kappa)>2$. The function is clearly positive for $t \in[1 / 2,1]$ if $\kappa \geq 1$. This is true since

$$
\kappa=\sqrt{-\hat{\mu}}>\sqrt{\pi}
$$

We calculate the first derivative

$$
\dot{f}(t)=\left(2 \pi-1+\kappa^{2}\right) \sin \pi t \cosh \kappa t+\left((\pi-2) \kappa-\frac{\pi}{\kappa}\right) \cos \pi t \sinh \kappa t
$$

which is positive on $(0,1 / 2)$ since both coefficients are, due to (33). Moreover, we get $\dot{f}(0)=0$ and $\ddot{f}(0)=\pi\left(2 \pi-1+\kappa^{2}\right)+\kappa\left((\pi-2) \kappa-\frac{\pi}{\kappa}\right)>0$ so that 0 is a local minimum and it follows that $f$ is strictly increasing on $(0,1 / 2)$. This finishes the proof of case 2 and of the index theorem 1.2 .

\section{Transversality}

3.1. Thom-Smale transversality. We recall the basics of Thom-Smale transversality theory. Let $\mathcal{A}, \mathcal{B}$ be smooth Banach manifolds which admit a countable atlas each and are modeled on separable Banach spaces. Let $\mathcal{E} \rightarrow \mathcal{A} \times \mathcal{B}$ denote a smooth Banach space bundle, $\mathcal{E}_{(a, b)}$ the fibre over $(a, b)$ and $\mathcal{F}$ a section of $\mathcal{E}$ of class $C^{\ell}, \ell \geq 1$. Define $\mathcal{F}_{b}(a)=\mathcal{F}_{a}(b)=\mathcal{F}(a, b)$ 
for $a \in \mathcal{A}, b \in \mathcal{B}$, and let $d$ denote the differential of a section followed by projection onto the fibre.

Theorem 3.1. Let $\mathcal{F}$ be a $C^{\ell}$-section of $\mathcal{E}$ as above and assume

$$
\begin{aligned}
& d \mathcal{F}_{b}(a): T_{a} \mathcal{A} \rightarrow \mathcal{E}_{(a, b)} \text { is Fredholm for all } a \in \mathcal{F}_{b}^{-1}(0) \text { and } \ell \geq \\
& \max \left\{1,1+\operatorname{Ind} d \mathcal{F}_{b}(a)\right\} \\
& d \mathcal{F}(a, b): T_{a} \mathcal{A} \times T_{b} \mathcal{B} \rightarrow \mathcal{E}_{(a, b)} \text { is surjective for all }(a, b) \in \mathcal{F}^{-1}(0)
\end{aligned}
$$

In this case the subset

$$
\left\{b \in \mathcal{B} \mid d \mathcal{F}_{b}(a) \text { is surjective for all } a \in \mathcal{F}_{b}^{-1}(0)\right\} \subset \mathcal{B}
$$

is residual and therefore dense.

Definition 3.2. Let $W$ be a closed subspace of a Banach space $X$. A closed subspace $V \subset X$ is called a topological complement of $X$, if $W \oplus V=X$ and $W \cap V=\{0\}$.

One crucial ingredient in the proof of theorem 3.1 is the following well known proposition. For the sake of completeness we give a proof following [Sa96].

Proposition 3.3. Let $X, Y, Z$ be Banach spaces. Assume $D: X \rightarrow Y$ is Fredholm and $L: Z \rightarrow Y$ is a bounded linear operator, then

i) the range of $D \oplus L: X \oplus Z \rightarrow Y$ is closed with a finite-dimensional complement

ii) if $D \oplus L$ is surjective, then $\operatorname{ker}(D \oplus L)$ admits a topological complement. Moreover, the projection on the second factor $\Pi: \operatorname{ker}(D \oplus L) \rightarrow Z$ is Fredholm with

$$
\text { ker } \Pi \simeq \operatorname{ker} D, \quad \text { coker } \Pi \simeq \operatorname{coker} D
$$

where coker $D:=Y /$ ran $D$ and similarly for coker $\Pi$.

Proof. ad $i$ ) Since ran $D$ is closed the quotient $Y /$ ran $D$ inherits the structure of a Banach space from $Y$ and the projection $p r: Y \rightarrow Y /$ ran $D$ is continous. By assumption $Y / \operatorname{ran} D$ is finite dimensional and so is its subspace $\operatorname{pr}(\operatorname{ran} L)$. Hence the latter space is closed and so is its preimage $p r^{-1}(\operatorname{pr}(\operatorname{ran} L))$ under the continous map $p r$, which is $\operatorname{ran} D+\operatorname{ran} L$. Finally, the complement of $\operatorname{ran}(D \oplus L)$ in $Y$ is contained in the finite dimensional space $Y / \operatorname{ran} D$.

ad $\boldsymbol{i i}$ ) It is well known, cf. [Br83] section II.4, that any finite dimensional subspace of a Banach space admits a topological complement. Let $X_{1}$ be a topological complement of ker $D$. Moreover, since $\operatorname{ran} D$ is closed with finite dimensional complement, we can write $Y=\operatorname{ran} D \oplus \operatorname{coker} D$. Surjectivity of $D \oplus L$ implies that coker $D \subset \operatorname{ran} L$ and so we may choose a basis $\left\{L z_{1}, \ldots, L z_{N}\right\}$ of coker $D$, where $\left\{z_{1}, \ldots, z_{N}\right\}$ is a set of linearly independent elements of $Z$. 
Now define the linear operator $T: \operatorname{ran} D \oplus \operatorname{coker} D \rightarrow \operatorname{ker} D \oplus X_{1} \oplus Z$

$$
T\left(y^{\prime}, y^{\prime \prime}\right)=\left(0, x^{\prime}, \sum_{\nu=1}^{N} \lambda_{\nu} z_{\nu}\right)
$$

where $x^{\prime}$ is determined uniquely by $y^{\prime}=D x^{\prime}$ and the coefficients $\lambda_{\nu}$ by $y^{\prime \prime}=\sum_{\nu=1}^{N} \lambda_{\nu} L z_{\nu}$. It is not hard to check that a topological complement of $\operatorname{ker}(D \oplus L)$ is given by ran $T$. Actually, since existence of a right inverse of a bounded linear operator is equivalent to surjectivity and existence of a topological complement of the kernel, we observe that $T$ is indeed a right inverse of $D \oplus L$.

Now assume $(x, z) \in \operatorname{ker}(D \oplus L)$, then

$$
(x, z) \in \operatorname{ker} \Pi \Leftrightarrow D x=0 \text { and } z=0 \Leftrightarrow(x, 0) \in \operatorname{ker} D \oplus\{0\}
$$

which proves that $\operatorname{ker} \Pi=\operatorname{ker} D \oplus 0$. Define

$$
L^{-1}(\operatorname{ran} D):=\{z \in Z \mid L z=D x \quad \text { for some } \quad x \in X\}
$$

then $\operatorname{ran} \Pi=L^{-1}(\operatorname{ran} D)$ and this set is closed: $\operatorname{ran} D$ is closed and so is its preimage under the continuous map $L$. Finally we obtain

$$
\operatorname{coker} \Pi=\frac{Z}{\operatorname{ran} \Pi}=\frac{Z}{L^{-1}(\operatorname{ran} D)} \simeq \frac{\operatorname{ran} L}{\operatorname{ran} D \cap \operatorname{ran} L}=\frac{Y}{\operatorname{ran} D}=\operatorname{coker} D
$$

where the first isomorphism is induced by $L$, namely

$$
\begin{aligned}
\frac{Z}{L^{-1}(\operatorname{ran} D)} & \rightarrow \frac{\operatorname{ran} L}{\operatorname{ran} D \cap \operatorname{ran} L} \\
{\left[z+L^{-1}(\operatorname{ran} D)\right] } & \mapsto[L z+\operatorname{ran} D \cap \operatorname{ran} L]
\end{aligned}
$$

and the subsequent identity is due to $Y=\operatorname{ran} D+\operatorname{ran} L$ and given by

$$
\begin{aligned}
\frac{\operatorname{ran} D+\operatorname{ran} L}{\operatorname{ran} D} & \rightarrow \frac{\operatorname{ran} L}{\operatorname{ran} D \cap \operatorname{ran} L} \\
{[L z+\operatorname{ran} D \cap \operatorname{ran} L] } & \mapsto[L z+\operatorname{ran} D]
\end{aligned}
$$

Since $d \mathcal{F}_{a}(b)$ is bounded $(\ell \geq 1)$ and $d \mathcal{F}(a, b)=d \mathcal{F}_{b}(a) \oplus d \mathcal{F}_{a}(b)$, part i) of proposition 3.3 tells that $\operatorname{ran} d \mathcal{F}(a, b)$ is closed. Therefore in order to verify $(S)$ it is sufficient to prove its density for all $(a, b) \in \mathcal{F}^{-1}(0)$. Now it is a consequence [Br83, cor. I.8] of the Hahn-Banach theorem that the latter is equivalent to its annihilator being trivial

$$
\left\{v^{*} \in \mathcal{E}_{(a, b)}^{*} \mid v^{*}(v)=0 \forall v \in \operatorname{ran} d \mathcal{F}(a, b)\right\}=\{0\} \quad \forall(a, b) \in \mathcal{F}^{-1}(0)
$$

In many applications it is more convenient to check conditions $(F)$ and $(A)$ instead of $(F)$ and $(S)$.

We sketch the proof of theorem 3.1: Properties $(F)$ and $(S)$ together with part $i$ i) of proposition 3.3 tell that for any $(a, b) \in \mathcal{F}^{-1}(0)$ its linearization $d \mathcal{F}(a, b)$ admits a topological complement and therefore a right inverse. This 
means, by definition, that 0 is a regular value of $\mathcal{F}$ and so, by the implicit function theorem, the so called universal moduli space

$$
X=\mathcal{F}^{-1}(0)
$$

is a $C^{\ell}$-Banach manifold. It is locally at $(a, b)$ modeled on the separable Banach space $\operatorname{ker} d \mathcal{F}(a, b)$ and admits a countable atlas. Define the projection onto the second factor $\pi: \mathcal{A} \times \mathcal{B} \supset X \rightarrow \mathcal{B}$ and observe that, by $i i$ ) of the Lemma,

$$
d \pi(a, b): \operatorname{ker}\left(d \mathcal{F}_{b}(a) \oplus d \mathcal{F}_{a}(b)\right) \rightarrow T_{b} \mathcal{B}, \quad(A, B) \mapsto B
$$

is Fredholm for any $(a, b) \in \mathcal{F}^{-1}(0)$ with

$$
\text { Ind } d \pi(a, b)=\operatorname{Ind} d \mathcal{F}_{b}(a)
$$

This means, again by definition, that $\pi$ is a Fredholm map of class $C^{\ell}$ between separable Banach manifolds. Finally we may apply the Sard-Smale theorem Sm73 to the countably many coordinate representations of $\pi$ and obtain that the set of regular values of $\pi$ is a residual subset of $\mathcal{B}$ in case $\ell \geq \max \left\{1,1+\operatorname{Ind} d \mathcal{F}_{b}(a)\right\}$. Now the theorem follows from the following well known result of Thom-Smale transversality theory

Lemma 3.4. Under the assumptions of theorem 3.1 it follows

$$
\{\text { regular values of } \pi\}=\left\{b \in \mathcal{B} \mid d \mathcal{F}_{b}(a) \text { is surjective } \forall a \in \mathcal{F}_{b}^{-1}(0)\right\}
$$

Proof. We have to show that for every $b \in \mathcal{B}$ it holds

$$
d \pi(a, b) \text { surjective } \forall a \in \mathcal{F}_{b}^{-1}(0) \quad \Longleftrightarrow \quad d \mathcal{F}_{b}(a) \text { surjective } \forall a \in \mathcal{F}_{b}^{-1}(0)
$$

Assume the right hand side was true and choose any $\hat{b} \in T_{b} \mathcal{B}$. By surjectivity of $d \mathcal{F}_{b}(a)$ we find $\hat{a} \in T_{a} \mathcal{A}$ such that $d \mathcal{F}_{b}(a) \hat{a}=d \mathcal{F}_{a}(b) \hat{b}$. Then the pair $(-\hat{a}, \hat{b}) \in T_{a} \mathcal{A} \times T_{b} \mathcal{B}$ is indeed element of $T_{(a, b)} X$ and is mapped to $\hat{b}$ under $d \pi(a, b)$.

To prove the other direction assume the left hand side was true, choose any $v \in \mathcal{E}_{(a, b)}$ and apply the surjectivity assumption $(S)$ of theorem 3.1 to conclude the existence of a pair $\left(\hat{a_{0}}, \hat{b}\right) \in T_{a} \mathcal{A} \times T_{b} \mathcal{B}$ being mapped to $v$ by $d \mathcal{F}(a, b)$; in other words

$$
d \mathcal{F}_{b}(a) \hat{a_{0}}+d \mathcal{F}_{a}(b) \hat{b}=v
$$

Given $\hat{b}$, surjectivity of $d \pi(a, b)$ implies existence of $\hat{a_{1}}$ such that $\left(\hat{a_{1}}, \hat{b}\right) \in$ $T_{(a, b)} X$, i.e. $d \mathcal{F}_{b}(a) \hat{a_{1}}+d \mathcal{F}_{a}(b) \hat{b}=0$. Now define $\hat{a}=\hat{a_{0}}-\hat{a_{1}}$ and observe that $d \mathcal{F}_{b}(a)\left(\hat{a_{0}}-\hat{a_{1}}\right)=v$.

3.2. Transversality in the $C^{k}$-category. In this subsection we fix $k \geq 2$ and apply Thom-Smale transversality theory to the following situation

$$
\mathcal{A}=W^{2,2}\left(S^{1}, M\right), \quad \mathcal{B}=C^{k}\left(S^{1} \times M, \mathbb{R}\right)
$$

The $C^{k-1}$-section $\mathcal{F}: \mathcal{A} \times \mathcal{B} \rightarrow \mathcal{E}$ is defined to be

$$
\mathcal{F}(x, V):=\operatorname{grad} \mathcal{S}_{V}(x)=-\nabla_{t} \dot{x}-\nabla V_{t}(x)
$$


The fibre of $\mathcal{E}$ at $(x, V)$ is $\mathcal{E}_{(x, V)}=L^{2}\left(S^{1}, x^{*} T M\right)$ and the linearization of $\mathcal{F}$ at a zero turns out to be

$$
\begin{gathered}
d \mathcal{F}(x, V): W^{2,2}\left(S^{1}, x^{*} T M\right) \times C^{k}\left(S^{1} \times M, \mathbb{R}\right) \rightarrow L^{2}\left(S^{1}, x^{*} T M\right) \\
(\xi, \hat{V}) \mapsto d \mathcal{F}_{V}(x) \xi+d \mathcal{F}_{x}(V) \hat{V}
\end{gathered}
$$

where

$$
\begin{aligned}
d \mathcal{F}_{V}(x) \xi & =-\nabla_{t} \nabla_{t} \xi-R(\xi, \dot{x}) \dot{x}-\nabla_{\xi} \nabla V_{t}(x)=A_{x} \\
d \mathcal{F}_{x}(V) \hat{V} & =-\nabla \hat{V}_{t}(x)
\end{aligned}
$$

Remark 3.5. 1) Consider the metric space $W^{2,2}\left(S^{1}, \mathbb{R}^{N}\right)$. It is separabel because the set of Fourier-series with coefficients in $\mathbb{Q}^{N}$ is countable and dense in $C^{\infty}\left(S^{1}, \mathbb{R}^{N}\right)$, which in turn is dense in $W^{2,2}\left(S^{1}, \mathbb{R}^{N}\right)$.

2) Recall that any metric space $X$ is paracompact, which by definition means that any open cover admits a locally finite refinement. If $X$ is in addition separable, we can conclude that any open cover admits a countable subcover. The following argument is taken from [Sa96]: if the cover $\left\{U_{\alpha}\right\}_{\alpha}$ is locally finite and $\left\{x_{i}\right\}_{i}$ is a dense sequence then the set of pairs $(\alpha, i)$ with $x_{i} \in U_{\alpha}$ is countable. Since every $U_{\alpha}$ contains some point $x_{i}$ the map $(\alpha, i) \mapsto \alpha$ is surjective.

3) For sufficiently large $N \in \mathbb{N}$ we can find an embedding $\iota: M \hookrightarrow \mathbb{R}^{N}$ and define $W^{2,2}\left(S^{1}, M\right)$ to be the set of those elements of $W^{2,2}\left(S^{1}, \mathbb{R}^{N}\right)$ whose image lies in $\iota(M)$. That way $W^{2,2}\left(S^{1}, M\right)$ inherits the metric from the ambient space and is therefore itself paracompact and separabel.

4) One can give $W^{2,2}\left(S^{1}, M\right)$ the structure of a Banach manifold modeled on the separabel Banach space $W^{2,2}\left(S^{1}, \mathbb{R}^{n}\right)$, where $n=\operatorname{dim} M$. An atlas can be constructed where the charts are labeled by smooth functions from $S^{1}$ to $M$. Since $W^{2,2}\left(S^{1}, M\right)$ is paracompact and separabel by 3$)$, we can apply 2) to get a countable subatlas.

Theorem 3.6. (Transversality in $C^{k}$ ) For every integer $k \geq 2$ the following are true:

i) The subset

$$
\mathcal{V}_{\text {reg }}^{k}=\left\{V \in C^{k}\left(S^{1} \times M, \mathbb{R}\right) \mid d \mathcal{F}_{V}(x) \text { is surjective } \forall x \in \mathcal{F}_{V}^{-1}(0)\right\}
$$

of the Banach space $\left(C^{k}\left(S^{1} \times M, \mathbb{R}\right),\|\cdot\|_{C^{k}}\right)$ is residual and therefore dense. ii) Fix $a \in \mathbb{R}$, then the set

$\mathcal{V}_{\text {reg }}^{k, a}=\left\{V \in C^{k}\left(S^{1} \times M, \mathbb{R}\right) \mid d \mathcal{F}_{V}(x)\right.$ is surjective $\left.\forall x \in \mathcal{F}_{V}^{-1}(0), \mathcal{S}_{V}(x)<a\right\}$

is open and dense in $\left(C^{k}\left(S^{1} \times M, \mathbb{R}\right),\|\cdot\|_{C^{k}}\right)$.

Proof. ad $i$ ) We prove that assumption $(F)$ holds. Let $x \in \mathcal{F}_{V}^{-1}(0)$. Recall that $d \mathcal{F}_{V}(x)$ is the perturbed Jacobi-operator $A_{x}$ and

$$
\operatorname{dim} \operatorname{ker} d \mathcal{F}_{V}(x)=\operatorname{dim} \operatorname{ker} A_{x}=\operatorname{Nullity}(x)<\infty
$$


by the Morse index theorem. Moreover, since $d \mathcal{F}_{V}(x)$ is selfadjoint

$$
\operatorname{coker} d \mathcal{F}_{V}(x) \simeq \operatorname{ker} d \mathcal{F}_{V}(x)
$$

This shows that $d \mathcal{F}_{V}(x)$ is Fredholm of index 0 for any $x \in \mathcal{F}_{V}^{-1}(0)$. Together with the assumption $k \geq 2$ it follows that the condition in Theorem 3.1 on the differentiability of $\mathcal{F}$ is satisfied: $k-1 \geq 1$.

It remains to verify assumption $(A)$, i.e. we have to show that

$$
\left\langle\eta, d \mathcal{F}_{V}(x) \xi\right\rangle=0 \quad \forall \xi \in W^{2,2}\left(x^{*} T M\right)
$$

and

$$
\left\langle\eta, d \mathcal{F}_{x}(V) \hat{V}\right\rangle=0 \quad \forall \hat{V} \in C^{k}\left(S^{1} \times M, \mathbb{R}\right)
$$

together imply $\eta=0$. The first condition says that $\eta \in \operatorname{ker} d \mathcal{F}_{V}(x)$. So it satisfies a linear second order ODE with coefficients of class $C^{k-2}$ and therefore $\eta \in C^{k}\left(x^{*} T M\right)$. Now assume by contradiction that there is $t_{0} \in S^{1}$ such that $\eta\left(t_{0}\right) \neq 0$. In five steps we are going to construct $\hat{V}_{t} \in C^{\infty}$ such that

$$
\left\langle\eta, \nabla \hat{V}_{t}(x)\right\rangle_{L^{2}}>0
$$

As our construction will be local, we may choose geodesic normal coordinates $\xi=\left(\xi^{1}, \ldots, \xi^{n}\right)$ around $x_{0}=x\left(t_{0}\right)$. Let $\iota$ denote the injectivity radius of $(M, g)$. The piece of the loop $x(t)$ which lies inside the coordinate patch determines the curve $\xi(t)$ via

$$
x(t)=\exp _{x_{0}} \xi(t)
$$

so that $\xi\left(t_{0}\right)=0$.

STEP 1 Because $x(t)$ is continuous, we may choose a constant $\delta_{1}>0$ sufficiently small such that

$$
|\xi(t)| \leq \iota / 2, \quad \forall t \in\left[t_{0}-\delta_{1}, t_{0}+\delta_{1}\right] .
$$

STEP 2 Because $\eta$ is continuous and $\eta\left(t_{0}\right) \neq 0$, we may choose a constant $\delta_{2}>0$ sufficiently small such that

$$
\left\langle\eta(t), \eta\left(t_{0}\right)\right\rangle>0, \quad \forall t \in\left[t_{0}-\delta_{2}, t_{0}+\delta_{2}\right] .
$$

STEP 3 Set $\delta=\min \left\{\delta_{1}, \delta_{2}\right\}$ and choose a cut-off function $\gamma \in C^{\infty}(\mathbb{R},[0,1])$ such that

$$
\gamma(t)= \begin{cases}1 & , t \in\left[t_{0}-\frac{\delta}{2}, t_{0}+\frac{\delta}{2}\right] \\ 0 & , t \notin\left[t_{0}-\delta, t_{0}+\delta\right] .\end{cases}
$$

STEP 4 Choose a cut-off function $\beta \in C^{\infty}(\mathbb{R},[0,1])$ such that

$$
\beta\left(|\xi|^{2}\right)= \begin{cases}1 & ,|\xi|^{2} \leq \iota^{2} / 2 \\ 0 & ,|\xi|^{2} \geq \iota^{2}\end{cases}
$$

STEP 5 We are ready to define $\hat{V}_{t}$

$$
\hat{V}_{t}(x)= \begin{cases}\gamma(t) \beta\left(|\xi|^{2}\right)\left\langle\eta\left(t_{0}\right), \xi\right\rangle & , x=\exp _{x_{0}} \xi \text { and }|\xi|^{2}<\iota^{2} \\ 0 & , \text { else. }\end{cases}
$$


Putting everything together we get

$$
\begin{aligned}
\left\langle\eta, \nabla \hat{V}_{t}(x)\right\rangle_{L^{2}} & =\int_{0}^{1}\left\langle\eta(t), \nabla \hat{V}_{t}(x(t))\right\rangle d t \\
& =\int_{0}^{1} d \hat{V}_{t}(x(t)) \circ \eta(t) d t \\
& =\left.\int_{\{t:|\xi(t)|<\iota\}} \frac{\partial \hat{V}_{t}}{\partial \xi^{j}}\right|_{\exp _{x_{0}} \xi(t)} \eta^{j}(t) d t \\
& =\int_{t_{0}-\delta}^{t_{0}+\delta}\left(2 \gamma(t) \beta^{\prime}\left(|\xi|^{2}\right)\langle\xi(t), \eta(t)\rangle\left\langle\eta\left(t_{0}\right), \xi(t)\right\rangle\right. \\
& =\int_{t_{0}-\delta}^{t_{0}+\delta} \gamma(t)\left\langle\eta\left(t_{0}\right), \eta(t)\right\rangle d t>0 .
\end{aligned}
$$

The third equality follows from the definition of $\hat{V}_{t}$ (Step 5), and the fourth one from Step $3(\operatorname{supp} \gamma)$ as well as a straight forward calculation. In the fifth equality we used that for $t \in\left[t_{0}-\delta, t_{0}+\delta\right]$ Step 1 implies $|\xi(t)|^{2} \leq \iota^{2} / 4$ and therefore, by Step $4, \beta^{\prime} \equiv 0$ and $\beta \equiv 1$. Step 2 gives the final strict inequality.

ad $i$ ) dense: Since

$$
\mathcal{V}_{r e g}^{k} \subset \mathcal{V}_{r e g}^{k, a}
$$

and $\mathcal{V}_{r e g}^{k}$ is a residual subset of $C^{k}\left(S^{1} \times M, \mathbb{R}\right)$ by $\left.i\right)$, the set $\mathcal{V}_{r e g}^{k, a}$ is too. By Baire's category theorem it is therefore dense.

open: We fix some regular $V$ and construct an open neighbourhood $W_{V}$ of $V$ in $C^{k}\left(S^{1} \times M, \mathbb{R}\right)$ such that $W_{V} \subset \mathcal{V}_{\text {reg. }}^{k, a}$. Recall that

$$
\pi: X=\mathcal{F}^{-1}(0) \rightarrow C^{k}\left(S^{1} \times M, \mathbb{R}\right)
$$

is a Fredholm map of class $C^{k-1}$. Since

$$
X^{a}=\left\{(x, v) \in X \mid \mathcal{S}_{V}(x)<a\right\}
$$

is open in $X$, it follows that the restriction

$$
\pi_{a}: X^{a} \rightarrow C^{k}\left(S^{1} \times M, \mathbb{R}\right)
$$

is Fredholm of class $C^{k-1}$ too. Now rewrite $\pi_{a}^{-1}(V)$ as

$$
\left\{x \in W^{2,2}\left(S^{1}, M\right) \mid d \mathcal{F}_{V}(x) \text { surjective, } \mathcal{S}_{V}(x)<a \forall x \in \mathcal{F}_{V}^{-1}(0)\right\} \times\{V\}
$$

and observe that this set is compact (cf. our remarks in the introduction on Cieliebak's uniform $C^{0}$-bound); in other words $\pi_{a}$ is a proper map. As will be discussed later surjectivity is an open condition and so there exists an open neighbourhood $U$ of $\pi_{a}^{-1}(V)$ in $X^{a}$ such that $d \mathcal{F}_{V^{\prime}}\left(x^{\prime}\right)$ is surjective for all $\left(x^{\prime}, V^{\prime}\right) \in U$. Now use continouity of $\pi_{a}$ and compactness of $\pi_{a}^{-1}(V)$ to conclude the existence of an open neighbourhood $W_{V}$ of $V$ in $C^{k}\left(S^{1} \times M, \mathbb{R}\right)$ such that $\pi_{a}^{-1}\left(W_{V}\right) \subset U$. It follows $W_{V} \subset \mathcal{V}_{\text {reg }}^{k, a}$ 
It remains to show that surjectivity of $d \mathcal{F}_{V}(x)$ is an open condition (in $X)$ : Note that for $\left(x^{\prime}, V^{\prime}\right)$ near $(x, V)$ the operators $d \mathcal{F}_{V}(x)$ and $d \mathcal{F}_{V^{\prime}}\left(x^{\prime}\right)$ (strictly speaking their representatives with respect to a trivialization of $\mathcal{E} \rightarrow W^{2,2} \times C^{k}$ at $\left.(x, V)\right)$ differ by a bounded operator, whose norm can be made arbitrarily small by choosing $\left(x^{\prime}, V^{\prime}\right)$ sufficiently close to $(x, V)$. In view of the subsequent lemma and the selfadjointness of the linear operators we get

$$
\begin{aligned}
& \text { dim coker } d \mathcal{F}_{V^{\prime}}\left(x^{\prime}\right)=\text { dim ker } d \mathcal{F}_{V^{\prime}}\left(x^{\prime}\right) \\
& \leq \operatorname{dim} \operatorname{ker} d \mathcal{F}_{V}(x) \\
& =\operatorname{dim} \text { coker } d \mathcal{F}_{V}(x)=0
\end{aligned}
$$

Lemma 3.7. Let $X, Y$ be Banach spaces and $D: X \rightarrow Y$ be a Fredholm operator. Then there exists an $\epsilon>0$ such that for any linear operator $L: X \rightarrow Y$ with $\|L\|<\epsilon$

$$
\operatorname{dim} \operatorname{ker}(D+L) \leq \operatorname{dim} \operatorname{ker} D
$$

Proof. Let $X_{1}$ be a topological complement of ker $D$. It suffices to show

$$
\operatorname{ker}(D+L) \cap X_{1}=\{0\}
$$

The restriction $\tilde{D}: X_{1} \rightarrow \operatorname{ran} D$ is a bounded bijection and therefore admits a bounded inverse $\tilde{D}^{-1}$ by the open mapping theorem. Let $x \in X_{1} \cap \operatorname{ker}(D+$ $L)$, i.e. $x=-\tilde{D} L x$, then

$$
\|x\|_{X}=\left\|\tilde{D}^{-1} L x\right\|_{X} \leq\left\|\tilde{D}^{-1}\right\| \cdot\|L\| \cdot\|x\|_{X} \leq \epsilon\left\|\tilde{D}^{-1}\right\| \cdot\|x\|_{X} .
$$

Choose $0<\epsilon<\left\|\tilde{D}^{-1}\right\|$, then it follows $x=0$.

\subsection{Transversality in the $C^{\infty}$-category.}

Proof. (of Theorem 1.1) ad $i$ ) $\mathcal{V}_{\text {reg }}^{a} \subset\left(C^{\infty}, d\right)$ dense: Given any $V \in$ $C^{\infty}\left(M \times S^{1}, \mathbb{R}\right)$ we have to construct a sequence $V_{k}^{\prime} \in \mathcal{V}_{r e g}^{a}$ such that

$$
\forall \epsilon>0 \quad \exists k_{0} \in \mathbb{N} \quad \forall k>k_{0} \quad: \quad d\left(V, V_{k}^{\prime}\right)<\epsilon .
$$

The idea will be to approximate $V$ by regular $V_{k}$ 's in the $C^{k}$-topology and then approximate $V_{k}$ by smooth regular elements $V_{k}^{\prime}$ in the $C^{k}$-topology. Finally we make use of the observation that in order to control the metric $d$ we essentially have to control only finitely many $C^{k}$-norms in its series, because the strong weights $1 / 2^{k}$ take care of all the other ones.

STEP 1 Because $\mathcal{V}_{\text {reg }}^{k, a}$ is dense in $\left(C^{k}\left(M \times S^{1}, \mathbb{R}\right),\|\cdot\|_{C^{k}}\right)$ for any integer $k \geq 2$, we can find $V_{k} \in \mathcal{V}_{\text {reg }}^{k, a}$ with $\left\|V-V_{k}\right\|_{C^{k}}<1 /(2 k)$. For $k=0,1$ let us define $V_{0}=V_{1}=V$.

STEP 2 Because $\mathcal{V}_{\text {reg }}^{k, a}$ is open in $\left(C^{k}\left(M \times S^{1}, \mathbb{R}\right),\|\cdot\|_{C^{k}}\right)$ for any integer $k \geq 2$, we can choose $0<\epsilon_{k}<1 /(2 k)$ sufficiently small such that $B_{\epsilon_{k}}\left(V_{k}\right)$, the open $\epsilon_{k}$-ball around $V_{k}$, is contained in $\mathcal{V}_{r e g}^{k, a}$. 
Step 3 Because $M \times S^{1}$ is compact, $C^{\infty}\left(M \times S^{1}, \mathbb{R}\right)$ is dense in $\left(C^{k}(M \times\right.$ $\left.\left.S^{1}, \mathbb{R}\right),\|\cdot\|_{C^{k}}\right)$ for $k \in \mathbb{N}_{0}$, cf. [Hi76, theorem 2.6]. Hence we can find $V_{k}^{\prime} \in C^{\infty} \cap B_{\epsilon_{k}}\left(V_{k}\right)$ for $k \geq 2$. For $k=0,1$ we define $V_{0}^{\prime}=V_{1}^{\prime}=V$.

STEP 4 Now pick $\epsilon>0$ and choose $\nu_{0} \in \mathbb{N}$ sufficiently large such that $f\left(\nu_{0}\right)=\sum_{\nu=\nu_{0}+1}^{\infty} 2^{-\nu}<\epsilon / 2$. Choose $k_{0}>\max \left\{\nu_{0}, 4 / \epsilon\right\}$ and observe that by Steps 1,2 and 3 for $k \geq 2$

$$
\left\|V-V_{k}^{\prime}\right\|_{C^{k}} \leq\left\|V-V_{k}\right\|_{C^{k}}+\left\|V_{k}-V_{k}^{\prime}\right\|_{C^{k}} \leq \frac{1}{2 k}+\epsilon_{k} \leq \frac{1}{k}
$$

Note that this implies $\left\|V-V_{k}^{\prime}\right\|_{C^{\nu}} \leq\left\|V-V_{k}^{\prime}\right\|_{C^{k}} \leq \frac{1}{k}$ for any $0 \leq \nu \leq k$. We get for any $k>k_{0}$

$$
\begin{aligned}
d\left(V, V_{k}^{\prime}\right) & =\sum_{\nu=0}^{\nu_{0}} \frac{1}{2^{\nu}} \frac{\left\|V-V_{k}^{\prime}\right\|_{C^{\nu}}}{1+\left\|V-V_{k}^{\prime}\right\|_{C^{\nu}}}+\sum_{\nu=\nu_{0}+1}^{\infty} \frac{1}{2^{\nu}} \frac{\left\|V-V_{k}^{\prime}\right\|_{C^{\nu}}}{1+\left\|V-V_{k}^{\prime}\right\|_{C^{\nu}}} \\
& \leq \sum_{\nu=0}^{\nu_{0}} \frac{1}{2^{\nu}} \frac{1}{k} \frac{1}{1+\left\|V-V_{k}^{\prime}\right\|_{C^{\nu}}}+\sum_{\nu=\nu_{0}+1}^{\infty} \frac{1}{2^{\nu}} \\
& \leq \frac{2}{k}+\frac{\epsilon}{2}<\epsilon .
\end{aligned}
$$

$\mathcal{V}_{\text {reg }}^{a} \subset\left(C^{\infty}, d\right)$ open: Pick $V \in \mathcal{V}_{\text {reg }}^{a}$ and set $k=2$. Exploiting openess of $\mathcal{V}_{\text {reg }}^{2, a}$ in $\left(C^{2}\left(M \times S^{1}, \mathbb{R}\right),\|\cdot\|_{C^{2}}\right)$ we are able to choose a constant $\epsilon_{0}>0$ such that for any $V^{\prime \prime}$ of class $C^{2}$ with $\left\|V-V^{\prime \prime}\right\|_{C^{2}}<\epsilon_{0}$ it follows $V^{\prime \prime} \in \mathcal{V}_{\text {reg }}^{2, a}$. Now define

$$
\epsilon=\frac{1}{4} \frac{\epsilon_{0}}{1+\epsilon_{0}}
$$

Let $V^{\prime}$ of class $C^{\infty}$ be such that $d\left(V, V^{\prime}\right)<\epsilon$. Therefore each term in the series on the left hand side has to be strictly smaller then $\epsilon$, in particular the second one

$$
\frac{1}{2^{2}} \frac{\left\|V-V^{\prime}\right\|_{C^{2}}}{1+\left\|V-V^{\prime}\right\|_{C^{2}}}<\epsilon=\frac{1}{4} \frac{\epsilon_{0}}{1+\epsilon_{0}} .
$$

But this is equivalent to

$$
\left\|V-V^{\prime}\right\|_{C^{2}}<\epsilon_{0}
$$

and therefore $V^{\prime} \in \mathcal{V}_{\text {reg. }}^{2, a}$. Finally $\mathcal{V}_{\text {reg }}^{a}=\mathcal{V}_{\text {reg }}^{2, a} \cap C^{\infty}\left(M \times S^{1}, \mathbb{R}\right)$ implies $V^{\prime} \in \mathcal{V}_{\text {reg }}^{a}$.

ad $\boldsymbol{i i})$ Since $\mathcal{V}_{r e g}^{a}$ is open and dense in $\left(C^{\infty}, d\right)$ it is residual. The identity

$$
\mathcal{V}_{r e g}=\bigcap_{a \in \mathbb{N}} \mathcal{V}_{r e g}^{a}
$$

implies the claim, because any countable intersection of residual sets is again residual and therefore dense. 


\section{Appendix A. Finite Sum}

For every $a \in \mathbb{R}$ and every $V \in \mathcal{V}_{\text {reg }}^{a}$, the set $C r i t$ is a 0 -dimensional manifold and the set $\mathrm{Crit}^{a}$ is finite. We recall that $\mathrm{Crit}^{a}$ consists by definition of the smooth maps $x: S^{1} \rightarrow M$ which satisfy

$$
-\nabla_{t} \dot{x}-\nabla V_{t}(x)=0
$$

as well as $\mathcal{S}_{V}(x)<a$. As a consequence the sum in (雨) is finite. The proof is standard and combines regularity theory, the implicit function theorem and compactness arguments.

Regularity. We need to extend the domain of definition of $\mathcal{S}_{V}$ to the Sobolev space $W^{2,2}\left(S^{1}, M\right)$ in order to apply the implicit function theorem in the next step. Our aim is to show that every $x \in W^{2,2}\left(S^{1}, M\right)$ which satisfies (35) almost everywhere is necessarily $C^{\infty}$-smooth. In view of the Sobolev embedding theorem we know that every $x \in W^{2,2}\left(S^{1}, M\right)$ is indeed of class $C^{1}$ and $\dot{x}$ is absolutely continous. If $x$ is in addition a solution to (35) we see that in local coordinates it holds almost everywhere

$$
\ddot{x}^{k}=-\Gamma_{i j}^{k}(x) \dot{x}^{i} \dot{x}^{j}-g^{k \ell}(x) \frac{\partial V_{t}}{\partial x^{\ell}}(x)
$$

Because the right hand side is of class $C^{0}$ and $\dot{x}$ is absolutely continous, it follows that $x$ is of class $C^{2}$. Hence the right hand side is $C^{1}$ and so $x$ is $C^{3}$. The iteration continues and we obtain finally $x \in C^{\infty}$.

Implicit function theorem. If $V \in \mathcal{V}_{\text {reg }}$, we know that zero is a regular value of the Fredholm section $\mathcal{F}$ in (34), which is of Fredholm index 0. Hence it follows from the infinite dimensional implicit function theorem that the zero set of $\mathcal{F}$ - which by regularity is precisely Crit - is a submanifold of dimension 0 of the domain $W^{2,2}\left(S^{1}, M\right)$. In particular this means that the elements of $C r i t$ are isolated.

Compactness. If $V \in \mathcal{V}_{r e g}^{a}$, then the set Crit $^{a}$ consists of finitely many elements: Let us assume by contradiction that it contains infinitely many distinct elements $\left\{x_{\nu}\right\}_{\nu \in \mathbb{N}}$. We prove that there exists $x \in$ Crit $^{a}$ and a subsequence $\left\{x_{\nu_{k}}\right\}_{k \in \mathbb{N}}$ converging to $x$ in $W^{2,2}\left(S^{1}, M\right)$, which is a contradiction to the former paragraph.

As we observed in section 2, an element $x_{\nu} \in$ Crit $^{a}$ corresponds to a 1-periodic Hamiltonian orbit $z_{\nu}=z_{x_{\nu}}=\left(x_{\nu}, g\left(x_{\nu}\right) \dot{x}_{\nu}\right)$ and we are going to prove that the uniform bound $a$ for $\mathcal{S}_{V}$ implies uniform bounds for the initial conditions $\left(x_{\nu}^{0}, y_{\nu}^{0}\right):=\left(x_{\nu}(0), g\left(x_{\nu}(0)\right) \dot{x}_{\nu}(0)\right) \in T^{*} M$. So the sequence of initial conditions lies in a compact subset of $T^{*} M$ and therefore admits a convergent subsequence $\left(x_{\nu_{k}}^{0}, y_{\nu_{k}}^{0}\right) \rightarrow\left(x_{0}, y_{0}\right) \in T^{*} M$ for $k \rightarrow \infty$. Let $\varphi_{t}: T^{*} M \rightarrow T^{*} M$ be the time- $t$-map of the Hamiltonian flow. The $\left(x_{\nu_{k}}^{0}, y_{\nu_{k}}^{0}\right)$ are fixed points of $\varphi_{1}$ and - because $\varphi_{1}$ is continous - so is $\left(x_{0}, y_{0}\right)$. In other words the limit $z(t)=\varphi_{t}\left(x_{0}, y_{0}\right)$ is a 1-periodic orbit. Setting $x(t)=\pi(z(t))$, where $\pi: T^{*} M \rightarrow M$ is the natural projection, we obtain that $x \in C$ rit $^{a}$. This shows that $x_{\nu_{k}} \rightarrow x$ in $C^{1}$. Moreover, using the fact that $x$ and $x_{\nu_{k}}$ both 
satisfy (35) this implies $x_{\nu_{k}} \rightarrow x$ in $C^{2}$. In view of the continous embedding $C^{2}\left(S^{1}, M\right) \hookrightarrow W^{2,2}\left(S^{1}, M\right)$ we obtain convergence in $W^{2,2}\left(S^{1}, M\right)$, but this contradicts the fact that the elements of Crit are isolated.

It remains to get the uniform bounds: Since $M$ is compact, there is nothing to prove for the base components $x_{\nu}(0) \in M$. Now the bound $a$ for the classical action $\mathcal{S}_{V}$ leads to a uniform $L^{2}$-bound for $\dot{x}$ for all $x \in W^{2,2}\left(S^{1}, M\right)$ with $\mathcal{S}_{V}(x)<a$, namely

$$
\|\dot{x}\|_{L^{2}}^{2}=\int_{0}^{1}|\dot{x}|^{2} d t<2 a+2\|V\|_{L^{\infty}\left(S^{1} \times M\right)}
$$

By (35), we get $\frac{d}{d t}\left|x_{\nu}\right|^{2}=-2\left\langle\nabla V_{t}\left(x_{\nu}\right), \dot{x}_{\nu}\right\rangle$ pointwise in $t$. Integrate this identity over the interval $[0, t]$ to obtain

$$
\left|\dot{x}_{\nu}(0)\right|^{2} \leq\left|\dot{x}_{\nu}(t)\right|^{2}+\|\nabla V\|_{L^{\infty}}^{2} \int_{0}^{1}\left|\dot{x}_{\nu}(\tau)\right|^{2} d \tau
$$

and hence by integrating again and using (36) it follows

$$
\left|\dot{x}_{\nu}(0)\right|^{2} \leq\left(1+\|\nabla V\|_{L^{\infty}}^{2}\right)\left\|\dot{x}_{\nu}\right\|_{L^{2}}^{2}<2\left(a+\|V\|_{L^{\infty}}\right)\left(1+\|\nabla V\|_{L^{\infty}}^{2}\right)
$$

where the right hand side only depends on $a$ and $V$.

\section{REFERENCES}

[Br83] Brezis H., Analyse fonctionelle - Thèorie et applications, Masson, Paris 1983.

[Ci94] Cieliebak K., Pseudo-holomorphic curves and periodic orbits on cotangent bundles, J. Math. Pures Appl. 73 (1994), 251-278.

[CZ84] Conley C., Zehnder E., Morse-type index theory for flows and periodic solutions for hamiltonian equations, Comm. Pure Appl. Math. XXXVII (1984), 207-253.

[DS94] Dostoglou S., Salamon D.A., Cauchy-Riemann operators, self-duality, and the spectral flow, in 'First European Congress of Mathematics' I, Invited Lectures (Part 1), Joseph A., Mignot F., Murat F., Prum B., Rentschler R. (editors), Birkhäuser Verlag, Progress in Mathematics 119 (1994), 511-545.

[Du76] Duistermaat J.J., On the Morse index in variational calculus, Advances in Math. 21 (1976), 173-195.

[Fl89] Floer A., Symplectic fixed points and holomorphic spheres Commun. Math. Phys. 120 (1989) 575-611.

[GL58] Gelfand I.M., Lidskii V.B., On the structure of the regions of stability of linear canonical systems of differential equations with periodic coefficients, Translations A.M.S. (2) 8 (1958), 143-181.

[Hi76] Hirsch M.W., Differential topology, Graduate Texts in Math. 33, Springer-Verlag New York 1976.

[RS80] Reed M., Simon B., Methods of modern mathematical physics, I Functional analysis, Academic Press 1980.

[RS93] Robbin J., Salamon D.A., The Maslov index for paths, Topology 32 (1993), 827844.

[RS95] Robbin J., Salamon D.A., The spectral flow and the Maslov index, Bull. London Math. Soc. 27 (1995), 1-33.

[Sa96] Salamon D.A., Spin geometry and Seiberg-Witten invariants, preliminary version June 1996.

[Sa99] Salamon D.A., Lectures on Floer Homology, in 'Symplectic Geometry and Topology', Eliashberg and Traynor (editors), IAS/Park City Mathematics series 7 (1999), 143-230. 
[Sm73] Smale S., An infinite dimensional version of Sard's theorem, Am. J. Math. 87 (1973), 213-221.

[SW01] Salamon D.A., Weber J., J-holomorphic curves in cotangent bundles and Morse theory on the loop space, in preparation.

[Vi90] Viterbo C., A new obstruction to embedding Lagrangian tori Invent. Math. 100 (1990), no. 2, 301-320.

[Vi96] Viterbo C., Functors and computations in Floer homology with applications, Part II, Preprint October 1996, revised February 1998.

[We99] Weber J., J-holomorphic curves in cotangent bundles and the heat flow, Dissertation TU Berlin, 1999.

[We01] Weber J., Geodesic homology, in preparation.

Departement Mathematik, ETH Zentrum, 8092 Zürich, Switzerland

E-mail address: joa@math.ethz.ch, http://www.math.sunysb.edu/ joa 